\title{
Direct numerical simulations of instability and boundary layer turbulence under a solitary wave
}

\author{
Celalettin E. Ozdemir ${ }^{1} \dagger$, Tian-Jian Hsu ${ }^{1}$ and S. Balachandar ${ }^{2}$ \\ ${ }^{1}$ Center for Applied Coastal Research, University of Delaware, Newark, DE 19716, USA \\ ${ }^{2}$ Mechanical \& Aerospace Engineering, University of Florida, Gainesville, FL 32611, USA \\ (Received 19 October 2012; revised 5 May 2013; accepted 11 July 2013; \\ first published online 28 August 2013)
}

A significant amount of research effort has been made to understand the boundary layer instability and the generation and evolution of turbulence subject to periodic/oscillatory flows. However, little is known about bottom boundary layers driven by highly transient and intermittent free-stream flow forcing, such as solitary wave motion. To better understand the nature of the instability mechanisms and turbulent flow characteristics subject to solitary wave motion, a large number of direct numerical simulations are conducted. Different amplitudes of random initial fluctuating velocity field are imposed. Two different instability mechanisms are observed within the range of Reynolds number studied. The first is a short-lived, nonlinear, longwave instability which is observed during the acceleration phase, and the second is a broadband instability that occurs during the deceleration phase. Transition from a laminar to turbulent state is observed to follow two different breakdown pathways: the first follows the sequence of $K$-type secondary instability of a near-wall boundary layer at comparatively lower Reynolds number and the second one follows a breakdown path similar to that of free shear layers. Overall characteristics of the flow are categorized into four regimes as: (i) laminar; (ii) disturbed laminar; (iii) transitional; and (iv) turbulent. Our categorization into four regimes is consistent with earlier works. However, this study is able to provide more specific definitions through the instability characteristics and the turbulence breakdown process.

Key words: coastal engineering, solitary waves, turbulent flows

\section{Introduction}

Fluid motion of highly transient and skewed characteristics is ubiquitous in nature. Internal waves due to strong tides in the vicinity of complex submarine terrains (Stastna \& Lamb 2002; Bogucki, Rodekopp \& Barth 2005; Diamessis \& Rodekopp 2006) are striking examples of this kind. Tsunami waves (e.g. Voit 1987) and skewed waves in shallow waters are other examples with many coastal engineering applications. Though highly idealized, many studies approximate such transient flow motion using solitary waves (Vittori \& Blondeaux 2011). Much of the literature on solitary wave motion is devoted to the dynamics of internal solitary wave motion in a

† Current Address: Applied Ocean Physics \& Engineering Department, Woods Hole Oceanographic Institution, 02543, Woods Hole, MA, USA. Email address for correspondence: cozdemir@whoi.edu 
stratified fluid environment (see for example Apel et al. 1975). In the past, tsunamirelated research has mostly focused on the tsunami wave generation, propagation, inundation, and optimum emergency plan development (Synolakis et al. 2008). Recent devastating tsunami events, such as the 2004 Indian Ocean tsunami and the 2011 Great Tohoku tsunami, have motivated researchers to further study the tsunami hazard in the built environment and the drastic change in the landform (Dawson \& Shi 2000). For example, to evaluate the structural stability of critical infrastructures, it becomes necessary to understand how the tsunami-induced nearbed/seabed processes can cause liquefaction, scour and deposition of sediment (e.g. Tonkin et al. 2003). Only recently has the boundary layer structure and turbulence under solitary wave motion been studied in detail (Liu \& Orfilia 2004; Liu, Park \& Cowen 2007; Sumer et al. 2010; Vittori \& Blondeaux 2008, 2011; Lin \& Rodekopp 2011; Blondeaux \& Vittori 2012). Moreover, the formation of coherent vortices close to the seabed and their dissipation may play an important role in the fate of the two-dimensional vortices shed from nearshore structures (e.g. Negretti \& Socolofsky 2005; Seol \& Jirka 2010; Son, Lynett \& Kim 2011). With these motivations, our aim is to augment our understanding of solitary-wave-driven bottom boundary layer turbulence.

Wave-induced motion of fluid in general, and that due to solitary waves in particular, is rich and complicated due to its time-dependent nature. Much of the complexity stems from the fact that the flow contains an isolated pair of acceleration and deceleration phases. While at acceleration a possible viscous instability might generate turbulence, more favourable conditions for turbulence generation are present at deceleration due to the adverse pressure gradient (Vittori \& Blondeaux 2008; Sumer et al. 2010). The adverse pressure gradient during the decelerating phase gives rise to the formation of an inflection point in the velocity profile, which is a necessary condition for the inviscid instability formation (Drazin \& Reid 1981). This can clearly be seen in figure $1(a)$, where the laminar non-dimensionalized velocity profiles (reproduced from Liu \& Orfilia 2004) of a wall-bounded solitary wave before, at, and after the peak far-stream velocity is shown. The wave starts at $\phi=-180^{\circ}$, ends at $\phi=180^{\circ}$, and the peak far-stream velocity occurs at $\phi=0^{\circ}$, and the pressure gradient is positive, zero, and negative, respectively. Shortly after the peak velocity (see $\phi=6^{\circ}$ in figure 1), the shear gradient $\left(\mathrm{d}^{2} U / \mathrm{d} y^{2}\right)$ within a thin layer close to the bottom boundary, $y=[0,0.3]$, is of opposite sign compared to the layer above $y=[0.3,4]$ (see figure $1 b$ ). This gives rise to a favourable condition for instability growth in the form of two counter-rotating vortices located above and below the velocity extremum.

Additional supporting evidence on turbulence generation at deceleration in the case of solitary wave motion over a wall boundary comes from the studies of Sumer et al. (2010), Vittori \& Blondeaux (2008, 2011), and Blondeaux, Pralits \& Vittori (2012) who observed turbulence only in the decelerating phase. Sumer et al. (2010) conducted a series of experiments in a U-tube for a range of Reynolds number. Based on their observations, they categorized the flow into four different regimes. In the laminar regime, the flow shows no sign of instability and turbulence. This regime is observed for $R e_{\delta}$ (see (2.14) in $\S 2$ for definition) less than 632. Between $R e_{\delta}=632$ and $R e_{\delta}=1000$, Sumer et al. (2010) categorized the flow as a laminar regime with vortex tubes and formation of spanwise rollers is observed. The third regime is categorized as the transitional regime for $R e_{\delta}$ greater than 1000, where the flow becomes turbulent over a portion of the wave period during deceleration, but remains laminar otherwise. Although not directly observed in their study due to limitations of the experimental facility, a plausible fourth regime was conjectured, where the flow is turbulent through the whole wave period. One point to note in the study of Sumer et al. (2010) is that 

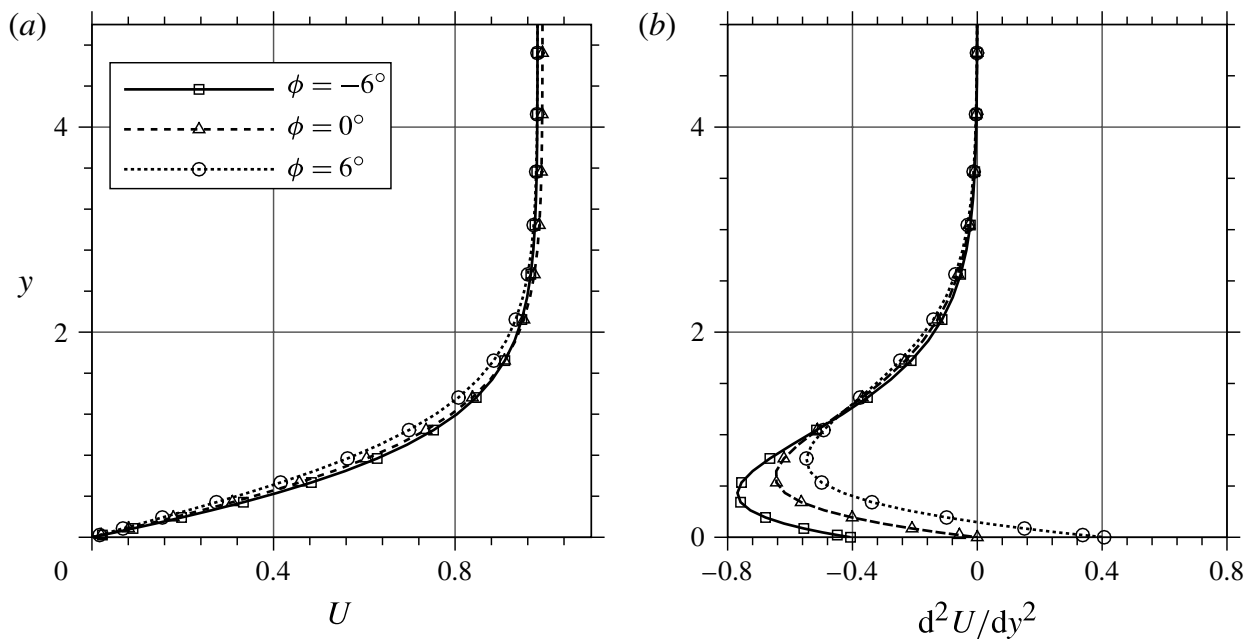

FIGURE 1. (a) Laminar velocity profiles of a solitary wave (Liu \& Orfilia 2004) at phases $\phi=-6^{\circ}, 0^{\circ}$ and $6^{\circ}$. (b) Shear gradient profiles at phases $\phi=-6^{\circ}, 0^{\circ}$ and $6^{\circ}$.

the experiments were designed such that the solitary wave is realized only between $\phi=-100^{\circ}$ and $\phi=100^{\circ}$ and the rollers observed in the laminar regime with vortex tubes are formed at around $\phi=100^{\circ}$. Therefore, the fate of the rollers in the laminar regime with vortex tubes, whether they decay, are sustained or grow through the whole wave period, remains an open question.

Vittori \& Blondeaux (2008, 2011) performed direct numerical simulations to understand the boundary layer turbulence under a solitary wave. They investigated boundary layer characteristics in a two-dimensional parametric space defined by the ratio of the viscous length scale to the flow depth and the ratio of free-surface wave amplitude to flow depth, defined by $a_{o}$. They categorized the flow into three regimes: (i) laminar; (ii) transitional; and (iii) turbulent.

In the recent theoretical study by Blondeaux et al. (2012), linear hydrodynamic stability analysis is performed for a single solitary wave of elevation. In this study, a momentary criterion for the onset of instability is introduced. The streamwise wavelength of maximum growth rate and the instant when the instability is initiated are compared with the experimental observations by Sumer et al. (2010) with very good agreement.

In the light of preceding studies, we are motivated to further study boundary layer instability and turbulence driven by solitary wave motion with specific emphasis on the initiation of instability, its dependence on initial perturbation and the pathway from initial instability to turbulence breakdown and dissipation. We conduct 70 direct numerical simulations at different levels of initial fluctuating velocity amplitude and Reynolds number but focus our investigation in this study on the limit of $a_{o}=0$. Hence, our numerical domain is similar to the experimental settings of Sumer et al. (2010). Although our domain is highly idealized and simulation conditions are only a subset of the full two-dimensional parametric space of Vittori \& Blondeaux (2008, 2011), special attention is given to instability mechanisms and the evolution of the transitional flow. The main findings are summarized here for the reader's convenience. We observed two possible instability mechanisms that might be present within a wave period. One is a nonlinear, viscous, long-wave instability that takes 
place at the acceleration phase if the background disturbance is of sufficient amplitude. This instability is observed to be short-lived and does not lead to chaotic motion at the Reynolds numbers considered. The second one is a broadband, linear instability at deceleration, which is responsible for the chaotic motion and transition to turbulence observed in this study. We also observed that the transition from laminar to nonlinear chaotic motion follows two different paths. The first path is similar to the $K$-type secondary instability of a boundary layer and it is observed at relatively lower $\operatorname{Re}_{\delta}$. The second one is similar to the transition route observed in free shear layers. Through comprehensive analyses, we can rigorously define and categorize the boundary layer flow driven by a solitary wave motion into four regimes: (i) laminar; (ii) disturbed laminar; (iii) transitional; and (iv) turbulent.

The rest of the paper is structured as follows. Section 2 gives the problem formulation and a brief description of numerical implementation. In $\S 3$, we present the qualitative and quantitative observations. Discussions on the instability mechanisms and the flow characteristics are presented in $\S 4$. In $\S 5$ findings are summarized and conclusions are presented.

\section{Problem formulation and numerical methodology}

\subsection{Problem formulation}

Before presenting the formulation of the present problem, it will be instructive to revisit the inviscid solution by Grimshaw (1971) and the viscous boundary layer solution of Liu \& Orfilia (2004) in the case of a flow driven by a solitary wave of elevation. The solitary wave solution of the Korteweg-deVries (KdV) equation is applicable to weakly nonlinear shallow flows. The weak nonlinearity is due to the solitary wave amplitude. Therefore, by using the perturbation approach with the Boussinesq approximation, Grimshaw (1971) was able to obtain the velocity profile of an inviscid flow driven by a solitary wave of elevation as an expansion in the small parameter $a_{o}$, which is the ratio of the wave amplitude, $\tilde{H}$, to the flow depth, $\tilde{h}$, as:

$$
\begin{aligned}
\frac{\tilde{U}(\tilde{y}, \tilde{t})}{\tilde{U}_{\infty o}}= & s^{2}-a_{o}\left[\left(-\frac{1}{4} s^{2}+s^{4}\right)+\left(\frac{\tilde{y}}{\tilde{h}}\right)^{2}\left(\frac{3}{2} s^{2}-\frac{9}{4} s^{4}\right)\right] \\
& +a_{o}^{2}\left[\left(\frac{19}{40} s^{2}+\frac{1}{5} s^{4}-\frac{6}{5} s^{6}\right)-\left(\frac{\tilde{y}}{\tilde{h}}\right)^{2}\left(-\frac{3}{2} s^{2}-\frac{15}{4} s^{4}+\frac{15}{2} s^{6}\right)\right. \\
& \left.-\left(\frac{\tilde{y}}{\tilde{h}}\right)^{4}\left(-\frac{3}{8} s^{2}-\frac{45}{16} s^{4}-\frac{45}{16} s^{6}\right)\right]+\cdots
\end{aligned}
$$

The tilde in the above equation and henceforth shows that the variable is dimensional. $\tilde{U}_{\infty o}$ is the maximum wave velocity and can be given as:

$$
\tilde{U}_{\infty o}=\frac{\tilde{H}}{\tilde{h}} \sqrt{g \tilde{h}}=a_{o} \sqrt{g \tilde{h}}, .
$$

where $g$ is the acceleration due to gravity. The term $s$ in (2.1) is given as follows:

$$
s=\operatorname{sech}[\tilde{\beta}(\tilde{x}-\tilde{c} \tilde{t})],
$$

where $\tilde{x}$ is the spatial location in the flow direction, $\tilde{c}$ is the wave speed of the solitary wave, and $\widetilde{\beta}$ is the wavenumber. In other words, the velocity of the solitary wave 
is given in the moving reference (i.e. in terms of $\tilde{x}-\tilde{c} \tilde{t}$ ). The wave speed and the wavenumber are given in terms of the wave amplitude as:

$$
\begin{gathered}
\tilde{c}=\sqrt{g \tilde{h}\left(1+a_{o}-\frac{1}{20} a_{o}^{2}-\frac{3}{70} a_{o}^{3}\right),} \\
\tilde{\beta}=\frac{1}{\tilde{h}} \sqrt{\frac{3 a_{o}}{4}}\left(1-\frac{5}{8} a_{o}+\frac{71}{128} a_{o}^{2}\right) .
\end{gathered}
$$

Here we will consider the orbital wavelength of the solitary wave to be much larger than the layer depth. Furthermore, we will investigate the behaviour of transition to turbulence locally within the boundary layer on a streamwise length scale much shorter than the length scale (orbital length) of the solitary wave. Thus, by setting $\tilde{x}=0,(2.3)$ becomes:

$$
s=\operatorname{sech}(-\widetilde{\beta} \tilde{c} \tilde{t})=\operatorname{sech}(-\widetilde{\omega} \tilde{t}),
$$

where $\widetilde{\omega}$ is the wave frequency. A positive (or negative) frequency corresponds to a right (or left) moving solitary wave, with corresponding positive (or negative) $\tilde{U}_{\infty o}$. The leading-order wave frequency is given as follows:

$$
\widetilde{\omega}=\frac{1}{\tilde{h}} \sqrt{\frac{3 a_{o}}{4} g \tilde{h}}
$$

In a sufficiently deep layer of fluid, if the thickness of the bottom boundary layer can be considered to be small compared to the length scale of vertical variation of the inviscid solution of the solitary wave given in (2.1), we can take the limit $\tilde{y} / \tilde{h} \rightarrow 0$ and obtain:

$$
\frac{\tilde{U}(\tilde{t})}{\tilde{U}_{\infty o}}=s^{2}-a_{o}\left(-\frac{1}{4} s^{2}+s^{4}\right)+a_{o}^{2}\left(\frac{19}{40} s^{2}+\frac{1}{5} s^{4}-\frac{6}{5} s^{6}\right) .
$$

It is clear that even sufficiently close to the bottom boundary, the velocity of the solitary wave is nonlinearly dependent on the ratio of wave amplitude to the flow depth, $a_{o}$. It should also be mentioned that both the maximum free-stream velocity $\tilde{U}_{\infty o}$ and the wave frequency $\widetilde{\omega}$ are dependent on $a_{o}$. Consequently, the exact nature of the bottom boundary layer instability and turbulence driven by the solitary wave is nonlinearly dependent on the solitary wave height.

The nonlinear effect of wave height was investigated in detail by Vittori \& Blondeaux (2011). The instability and boundary layer turbulence characteristics in Utube experiments conducted by Sumer et al. (2010) can be considered to approximate the limit $a_{o} \rightarrow 0$. Good agreement between the U-tube experiments and the direct numerical simulation results was reported (Vittori \& Blondeaux 2011).

A leading-order viscous extension of Grimshaw's (1971) inviscid solution for the laminar boundary layer driven by a solitary wave was obtained by Liu \& Orfilia (2004). In the following study of Liu et al. (2007), the second-order terms in the governing partial differential equation are also included and nearly identical results to the ones in Liu \& Orfilia (2004) are obtained. Figure 2(a) shows the time history of the wave velocity outside the bottom boundary layer. In the range $a_{o}=[0,0.3]$ a deviation in far-field velocity can be observed between $\phi \approx-50^{\circ}$ and $\phi \approx 50^{\circ}$. The influence of wave height can further be observed in the laminar velocity profiles. The deviation of the velocity profiles for $a_{o}>0$ from those of $a_{o}=0$ becomes maximum 

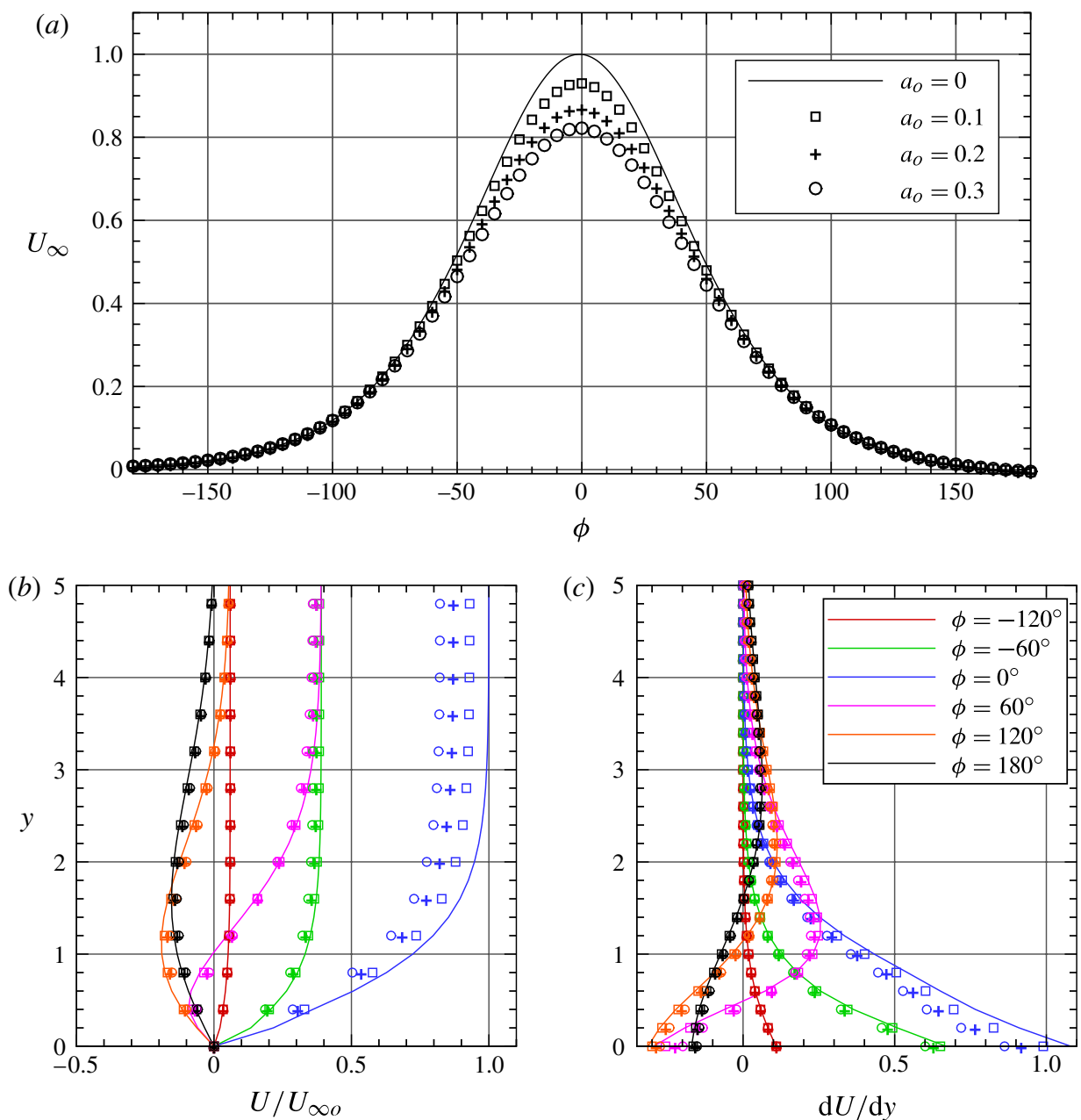

FIgURE 2. (a) Time series of free-stream velocity in a wave period, $(b)$ laminar velocity profiles (Liu \& Orfilia 2004), and (c) laminar shear profiles (Liu \& Orfilia 2004), obtained for the first (solid line) and third order of Grimshaw's (1971) solution (symbols). On each plot, open squares $(\square)$, plus signs $(+)$, and open circles $(\bigcirc)$ show $a_{o}=0.1, a_{o}=0.2, a_{o}=0.3$, respectively.

at $\phi=0^{\circ}$ and remains noticeable in the range $\phi=\left[-50^{\circ}, 50^{\circ}\right]$. In addition, the peak deviation is at the top of the boundary layer (figure $2 b$ ). Further investigation of shear profiles for $a_{o}>0$ shows that the shear profiles are qualitatively close to those of $a_{o}=0$ and the effect of finite $a_{o}$ is to reduce the shear within the boundary layer figure $2(c)$.

The direct numerical simulations of Vittori \& Blondeaux (2011) considered the influence of both $a_{o}$ and the non-dimensional boundary layer thickness $\left(\delta^{*}\right)$ normalized by the layer depth. In this study, we simplify the problem by considering the limit of $a_{o} \rightarrow 0$ and consider variations in boundary layer thickness, specified in terms of boundary layer Reynolds number (to be defined below). However, for each Reynolds number we consider various initial disturbance amplitudes to investigate possible nonlinear instability. Thus the present study complements the investigation of Vittori \& Blondeaux (2011). 
We assume that the flow is incompressible and hence the velocity vector is divergence free:

$$
\widetilde{\nabla} \cdot \widetilde{\boldsymbol{U}}=0
$$

We assume a solitary wave boundary layer driven by the following free-stream velocity:

$$
\tilde{U}_{\infty}=\tilde{U}_{\infty o} \operatorname{sech}^{2}(-\widetilde{\omega} \tilde{t}), \quad-\pi=-180^{\circ} \leqslant \widetilde{\omega} \tilde{t} \leqslant \pi=180^{\circ} .
$$

Here, $\phi=-180^{\circ}$ corresponds to the start and $\phi=180^{\circ}$ corresponds to the end of the solitary wave. Above the boundary layer, the viscous stress becomes unimportant in the streamwise $(\tilde{x})$ direction and hence the momentum equation and the time rate of change in the streamwise velocity gives the streamwise pressure gradient as:

$$
-\frac{1}{\rho} \frac{\partial \tilde{P}}{\partial \tilde{x}}=2 \tilde{U}_{\infty o} \widetilde{\omega} \operatorname{sech}^{2}(-\widetilde{\omega} \tilde{t}) \tanh (-\widetilde{\omega} \tilde{t}) .
$$

After substituting the mean streamwise pressure gradient into the momentum equation for the bottom boundary layer, the following dimensional form is obtained:

$$
\frac{\widetilde{\mathrm{D}} \widetilde{\boldsymbol{U}}}{\widetilde{\mathrm{D}} \tilde{t}}=2 \tilde{U}_{\infty o} \widetilde{\omega} \operatorname{sech}^{2}(-\widetilde{\omega} \tilde{t}) \tanh (-\widetilde{\omega} \tilde{t}) \boldsymbol{e}_{1}+\frac{1}{\rho} \widetilde{\nabla} P^{\prime}+\nu \widetilde{\boldsymbol{\nabla}}^{2} \widetilde{\boldsymbol{U}}
$$

where $\boldsymbol{e}_{1}$ is the unit vector that points along the positive streamwise flow direction $(x$ direction), and $P^{\prime}$ is the dynamic pressure. The momentum equation given in (2.12) is further non-dimensionalized by using $\tilde{U}_{\infty o}$ as the velocity scale, the Stokes laminar boundary layer thickness, $\widetilde{\delta}=\sqrt{2 v / \widetilde{\omega}}$, as the length scale, and $\widetilde{\delta} / \tilde{U}_{\infty o}$ as the time scale. The resulting non-dimensional momentum equation is written as:

$$
\frac{\mathrm{D} \boldsymbol{U}}{\mathrm{D} t}=\frac{4}{R e_{\delta}} \operatorname{sech}^{2}\left(\frac{-2 t}{R e_{\delta}}\right) \tanh \left(\frac{-2 t}{R e_{\delta}}\right) \boldsymbol{e}_{1}+\nabla P^{\prime}+\frac{1}{R e_{\delta}} \nabla^{2} \boldsymbol{U},
$$

in which $R e_{\delta}$ is the Reynolds number based on the Stokes thickness and the peak free-stream velocity:

$$
\operatorname{Re}_{\delta}=\frac{\tilde{U}_{\infty o} \tilde{\delta}}{v}
$$

The above Reynolds number can be expressed in terms of the two parameters employed by Vittori \& Blondeaux (2011) as:

$$
\operatorname{Re}_{\delta}=\frac{a_{o}^{3 / 4} \tilde{h}}{\delta^{*}}\left(\frac{64}{3}\right)^{1 / 4}
$$

Note that the definition of dimensional boundary layer thickness used by Vittori \& Blondeaux (2011) differs from $\widetilde{\delta}$. Also, in this study as $a_{o} \rightarrow 0$ the boundary layer thickness $\delta^{*} \rightarrow 0$ as well, resulting in finite Reynolds number.

Vittori \& Blondeaux (2011) discuss the range of possible values for these parameters as follows:

$$
\begin{gathered}
0.05<a_{o}<0.7 \\
2.8 \times 10^{-4}<\left(\frac{3 a_{o}}{4}\right)^{-1 / 4} \frac{\tilde{\delta}}{\tilde{h}}<1.34 \times 10^{-3} .
\end{gathered}
$$


From the above equations the upper limit of Reynolds number for any given $\epsilon$ can easily be calculated. For example, if $a_{o}=0.3$, the peak Reynolds number can be calculated as 3110 , which is acceptably close to the largest of the Reynolds numbers considered in this study.

\subsection{Numerical methodology}

The governing equations are solved in a computational domain of a plane channel. The channel is of $80 \times 80 \times 40$ non-dimensional units along the $x, y$ and $z$ directions, respectively, where $x$ is the positive flow direction, $y$ is the vertical direction and $z$ is the spanwise direction. At the top and the bottom planes, no-slip and no-penetration wall boundary conditions are imposed. In the edge planes in the $x$ and $z$ directions, periodic boundary conditions are implemented. In this study, we investigate the instability mechanisms and the characteristics of turbulence due to an isolated solitary wave motion. Therefore, sensitivity of the flow characteristics to the amplitude of initial velocity fluctuations must be considered. To carefully investigate the influence of the initial disturbance, we have implemented five levels of random background disturbance and its form is given as follows:

$$
\begin{aligned}
& \boldsymbol{U}^{\prime}\left(x, y, z, \phi=-180^{\circ}\right)=\wp\left(\varepsilon f \boldsymbol{e}_{1}\right), \\
& \varepsilon=\{0.01,0.02,0.05,0.10,0.20\},
\end{aligned}
$$

where $f$ is a function that generates random numbers with a uniform probability distribution between -1.0 and 1.0 and $\varepsilon$ is the amplitude factor that controls the magnitude of the random vector field. In the above equation, note that the initial fluctuation velocity field, $f \boldsymbol{e}_{1}$, does not satisfy the divergence-free condition, and thus the operator $\wp$ projects the initial fluctuations to the divergence-free space. Note that the projection operator is linear and does not affect the relative amplitude of the different initial magnitudes of the disturbance. The magnitude of the random initial background disturbance is chosen to be at five different levels between $1 \%$ and $20 \%$. In other words, for a given $R e_{\delta}$, with the same distribution of fluctuating velocity field, five independent simulations with different magnitudes of initial disturbance are conducted. At this point, it should be mentioned that a disturbance amplitude of 0.2 may be considered as large when investigating the hydrodynamic instability. On the other hand, as to be presented in $\S 3$, any disturbance at $\phi=-180^{\circ}$ undergoes a huge decay in the first half of the acceleration. Therefore, the amplitude of $\varepsilon=0.2$ at $\phi=-180^{\circ}$ is equivalent to a much smaller amplitude before any instability is observed in the simulations conducted.

Highly accurate pseudo-spectral schemes are used to solve the governing equations. The computational domain is discretized in the $x$ and $z$ directions by equally spaced grid points and the variables to be solved are expanded in Fourier space. For the non-homogeneous $y$ direction, non-uniform Chebyshev-Gauss-Lobatto grid points are employed and the variables are expanded in Chebyshev space (Canuto et al. 1987). The number of grid points is assigned to be 128, 193, and 128 in the $x, y$ and $z$ directions, respectively. The time integration is computed through a hybrid method, where the explicit Crank-Nicolson scheme is used for the diffusion terms and the lowstorage third-order-accurate Runge-Kutta method otherwise (for details see Cortese \& Balachandar 1995 and Cantero et al. 2008).

As to be presented in $\S 3.1$, the domain size is sufficiently large to capture the largest eddies generated as a result of linear hydrodynamic instabilities. The computational domain captures six spanwise rollers which is also in accordance with the previous experimental and numerical observations (see Sumer et al. 2010; Vittori \& 
Blondeaux 2011). In the turbulence phase of the flow, when it exists, the streamwise two-point correlation of velocity was computed and it decayed sufficiently along the streamwise direction to confirm the adequacy of the computational domain (not shown). The grid size is chosen to be small enough to resolve the smallest dissipative scales. In particular, at each Reynolds number, based on the largest wall shear stress, the grid resolution in each of the three directions can be evaluated in terms of wall units. The maximum grid spacing (when $R e_{\delta}=5000$ ) in the streamwise and spanwise direction is $\Delta x^{+}=30$ and $\Delta z^{+}=15$, respectively. In the inhomogeneous wall-normal direction, the minimum grid spacing, $\Delta y_{\text {min }}^{+}$, is about 0.4 , and the maximum grid spacing, $\Delta y_{\max }^{+}$, is around 40 at the mid-vertical plane. Furthermore, it has been verified that even for the case of most intense turbulence (case R5000-0.20) five grid points exist within the viscous sub-layer. These grid spacings are consistent with the resolution requirements set forth in the turbulent channel flow simulations under both stationary and oscillatory conditions (Kim, Moin \& Moser 1987; Spalart \& Baldwin 1989). Furthermore, from the decay of the energy spectra of the velocity fluctuations over several decades of dissipation it can be concluded that the resolution is sufficiently fine to capture the smallest turbulent length scales. More discussion on the energy spectra of the velocity components for the different Reynolds numbers is given in $\S 4$.

\section{Results}

\subsection{Vortex structures}

To illustrate the different turbulent and transitional characteristics, which lead to our final remarks on the four flow regimes, we select three representative simulation results among the 70 simulations performed. A complete list of all the cases simulated is shown in table 1. The naming convention for the simulated cases is that the letter ' $\mathrm{R}$ ' is followed by Reynolds number, $R e_{\delta}$, which is then followed by the amplitude factor $\varepsilon$ of the initial perturbation. The three cases to be discussed here are R15000.20 , R2000-0.20, and R2500-0.01. Along with the complete laminar regime, these three cases represent typical vortex structures for disturbed laminar, transitional and turbulent regimes, respectively.

Figure 3 shows the two-dimensional vortex structures at $z=10$ for case R15000.20 (i.e. $R e_{\delta}$ is equal to 1500 and the amplitude factor $\varepsilon$ of the initial fluctuating velocity is 0.2 ). Vortex structures are identified by $\lambda_{c i}$, a measure of the local swirling strength, which is calculated as the imaginary part of the complex eigenvalues of the velocity gradient tensor (Zhou et al. 1999). We choose to present $\lambda_{c i}$ contours on a selected $x-y$ plane, instead of three-dimensional iso-contours of $\lambda_{c i}$, due to the fact that $\lambda_{c i}$ values over the entire computational domain are comparable in magnitude during most of the wave period. Therefore, any growth in swirling strength close to the bottom wall cannot be easily distinguished in the three-dimensional view. The growth of two-dimensional vortex structures is first observed at around $\phi=-60^{\circ}$ (see figure $3 a$ ). Two-dimensional quasi-circular vortices, which are randomly distributed in the $x$ direction can be observed within $y=0$ to 1 . The swirling strength of these vortices is smaller than the ones above $y=1$ which are the remnants of the initial fluctuations. In addition to their random locations, the maximum swirling strength in the core of these structures also shows variation. From $\phi=-24^{\circ}$ to $0^{\circ}$, these structures change their location not only in the $x$ direction due to advection, but also in the vertical $(y)$ direction. Compared to the swirling strength at $\phi=-60^{\circ}$, some of these coherent structures lose their strength, some of them stay the same and others 


\begin{tabular}{|c|c|c|c|}
\hline Case name & $R e_{\delta}$ & $\varepsilon$ & Regime \\
\hline R400-0.01 & 400 & 0.01 & Laminar \\
\hline R400-0.02 & & 0.02 & Laminar \\
\hline R400-0.05 & & 0.05 & Laminar \\
\hline R400-0.10 & & 0.10 & Laminar \\
\hline R400-0.20 & & 0.20 & Laminar \\
\hline R500-0.01 & 500 & 0.01 & Laminar \\
\hline R500-0.02 & & 0.02 & Laminar \\
\hline R500-0.05 & & 0.05 & Laminar \\
\hline R500-0.10 & & 0.10 & Laminar \\
\hline R500-0.20 & & 0.20 & Dist. laminar \\
\hline R600-0.01 & 600 & 0.01 & Laminar \\
\hline R600-0.02 & & 0.02 & Laminar \\
\hline R600-0.05 & & 0.05 & Laminar \\
\hline R600-0.10 & & 0.10 & Dist. laminar \\
\hline R600-0.20 & & 0.20 & Dist. laminar \\
\hline R1000-0.01 & 1000 & 0.01 & Dist. laminal \\
\hline R1000-0.02 & & 0.02 & Dist. laminar \\
\hline R1000-0.05 & & 0.05 & Dist. laminar \\
\hline R1000-0.10 & & 0.10 & Dist. laminar \\
\hline R1000-0.20 & & 0.20 & Dist. lamina \\
\hline R1500-0.01 & 1500 & 0.01 & Dist. lamina \\
\hline R1500-0.02 & & 0.02 & Dist. lamina \\
\hline R1500-0.05 & & 0.05 & Dist. laminar \\
\hline R1500-0.10 & & 0.10 & Dist. laminar \\
\hline R1500-0.20 & & 0.20 & Dist. laminar \\
\hline R2000-0.01 & 2000 & 0.01 & Transitional \\
\hline R2000-0.02 & & 0.02 & Transitional \\
\hline R2000-0.05 & & 0.05 & Transitional \\
\hline R2000-0.10 & & 0.10 & Transitional \\
\hline R2000-0.20 & & 0.20 & Transitional \\
\hline R2100-0.01 & 2100 & 0.01 & Transitional \\
\hline R2100-0.02 & & 0.02 & Transitional \\
\hline R2100-0.05 & & 0.05 & Transitional \\
\hline R2100-0.10 & & 0.10 & Transitional \\
\hline R2100-0.20 & & 0.20 & Transitional \\
\hline R2200-0.01 & 2200 & 0.01 & Transitional \\
\hline R2200-0.02 & & 0.02 & Transitional \\
\hline R2200-0.05 & & 0.05 & Transient \\
\hline R2200-0.10 & & 0.10 & Turbulent \\
\hline R2200-0.20 & & 0.20 & Turbulent \\
\hline R2400-0.01 & 2400 & 0.01 & Turbulent \\
\hline R2400-0.02 & & 0.02 & Turbulent \\
\hline R2400-0.05 & & 0.05 & Turbulent \\
\hline R2400-0.10 & & 0.10 & Turbulent \\
\hline R2400-0.20 & & 0.20 & Turbulent \\
\hline R2500-0.01 & 2500 & 0.01 & Turbulent \\
\hline R2500-0.02 & & 0.02 & Turbulent \\
\hline R2500-0.05 & & 0.05 & Transitional \\
\hline R2500-0.10 & & 0.10 & Turbulent \\
\hline R2500-0.20 & & 0.20 & Turbulent \\
\hline
\end{tabular}

TABLE 1. (Continued on next page) 


\begin{tabular}{|c|c|c|c|}
\hline Case name & $R e_{\delta}$ & $\varepsilon$ & Regime \\
\hline R3000-0.01 & 3000 & 0.01 & Turbulent \\
\hline R3000-0.02 & & 0.02 & Turbulent \\
\hline R3000-0.05 & & 0.05 & Turbulent \\
\hline R3000-0.10 & & 0.10 & Turbulent \\
\hline R3000-0.20 & & 0.20 & Turbulent \\
\hline R3500-0.01 & 3500 & 0.01 & Turbulent \\
\hline R3500-0.02 & & 0.02 & Turbulent \\
\hline R3500-0.05 & & 0.05 & Turbulent \\
\hline R3500-0.10 & & 0.10 & Turbulent \\
\hline R3500-0.20 & & 0.20 & Turbulent \\
\hline R4000-0.01 & 4000 & 0.01 & Turbulent \\
\hline R4000-0.02 & & 0.02 & Turbulent \\
\hline R4000-0.05 & & 0.05 & Turbulent \\
\hline R4000-0.10 & & 0.10 & Turbulent \\
\hline R4000-0.20 & & 0.20 & Turbulent \\
\hline R5000-0.01 & 5000 & 0.01 & Turbulent \\
\hline R5000-0.02 & & 0.02 & Turbulent \\
\hline R5000-0.05 & & 0.05 & Turbulent \\
\hline R5000-0.10 & & 0.10 & Turbulent \\
\hline $5000-0.20$ & & 0.20 & Turbulent \\
\hline
\end{tabular}

TABLE 1. List of all the simulation cases. The nomenclature is as follows: the number following $\mathrm{R}$ indicates the Reynolds number and the number following the hyphen denotes the amplitude of the initial velocity fluctuations. The regimes observed as a result are also listed as: (i) laminar, (ii) disturbed laminar, (iii) transitional, and (iv) turbulent (See $\$ 4.4$ for details)

increase. The increasing and decreasing swirling strength in these structures seems to be closely affected by the swirling strength of the vortex structures just above them. The vortex structures continue to move in the $y$ direction slowly and are located between $y=[1,3]$ at $\phi=90^{\circ}$. At this instant, it is important to note that the secondary rollers are triggered between the bottom wall and $y=1$. From $\phi=90^{\circ}$ to $\phi=180^{\circ}$, the swirling strength of these near-bottom rollers becomes more intense, together with that of the rollers above them. At $\phi=180^{\circ}$ a pair of rollers in the $y$-direction, very organized in shape and distributed almost equally spaced in the streamwise direction can be observed.

There are two striking observations on the overall vortex formation and evolution. First, at $\phi=90^{\circ}$ the swirling strength of the upper rollers is one order of magnitude smaller compared with the ones at $\phi=0^{\circ}$ (notice different levels of contours defined). However, they are able to trigger vortices at the bottom wall. This observation is closely related to the temporal change in the location of the inflection and extremum points in the velocity profile (see figure $1 b$ ), which is critical to the generation of organized flow structures and the sustainability of these structures. This point will be discussed in more detail in $\$ 3.2$. The second observation to be noted is at $\phi=120^{\circ}$ there are six rollers close to the bottom wall, but only four of them grow and remain at the end of the wave period, i.e. $\phi=180^{\circ}$.

At $R e_{\delta}=2000$ (case R2000-0.20, shown in figure 4), coherent vortices are first observed from three-dimensional $\lambda_{c i}^{2}$ iso-contours at around $\phi=100^{\circ}$ (not shown). In figure $4(a)$, at $\phi=114^{\circ}$ the initial formation of the vortex tubes which are aligned 
(a)

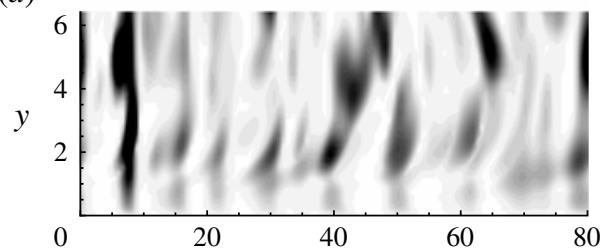

(c)

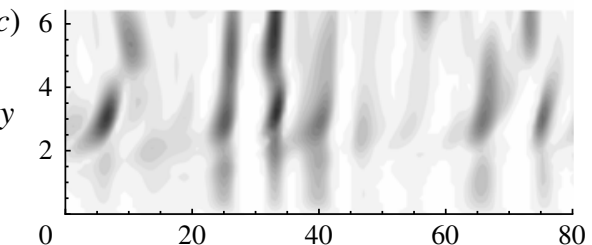

(e)
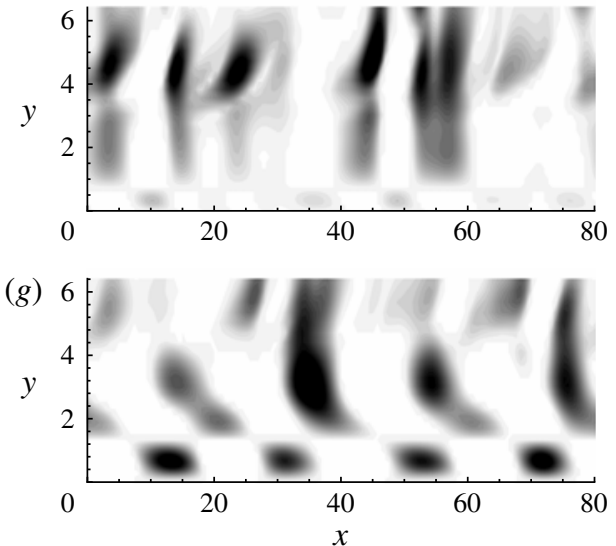

(b)

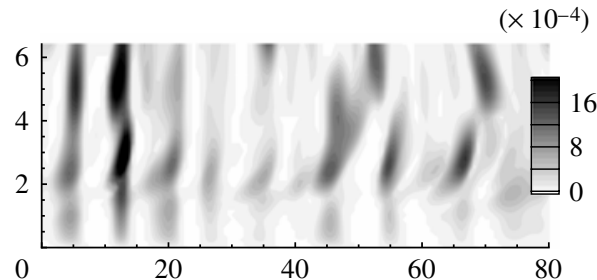

(d)

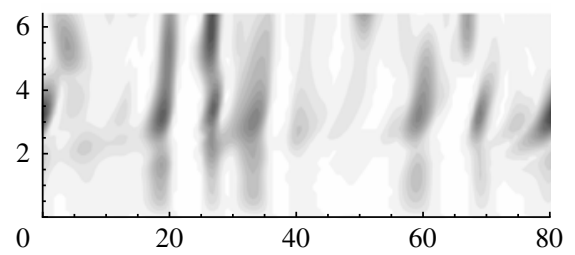

$(f)$

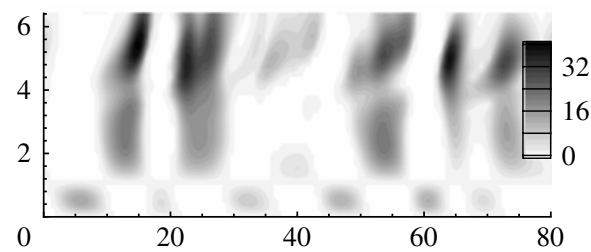

(h)

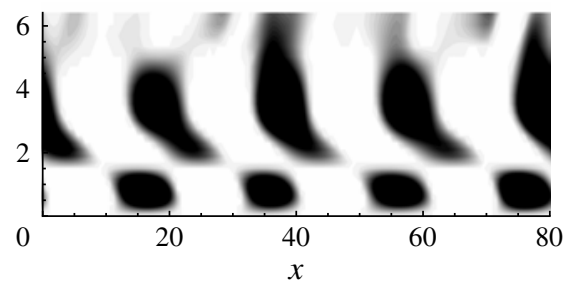

FIgURE 3. Contours of $\lambda_{c i}^{2}$ at $z=10$ for case R1500-0.20 at phases: $(a) \phi=-60^{\circ}$; $(b)$ $\phi=-24^{\circ} ;(c) \phi=-12^{\circ} ;(d) \phi=0^{\circ} ;(e) \phi=90^{\circ} ;(f) \phi=120^{\circ} ;(g) \phi=150^{\circ} ;(h) \phi=180^{\circ}$. The levels of the contours for $(a-d)$ are given in $(b)$, and the levels of contours $(e-h)$ are given in $(f)$.

in the spanwise direction is clearly observed. There are six spanwise vortex tubes that can be identified that are equally spaced along the streamwise direction, and the distance between two consecutive rollers is approximately 13.34. The observed distance between consecutive rollers is nearly identical to that of Sumer et al. (2010) at $R e_{\delta}=875$. However, since initial conditions differ between the simulations and experiments caution should be exercised. Although there is a slow increase in the swirling strength starting from the instant they form, the amount of increase is not sufficient to initiate a nonlinear growth until $\phi=144^{\circ}$ (see figure $4 b$ ). At $\phi=156^{\circ}$, the rollers start to deform into $\Lambda$-shaped vortices. At $\phi=159^{\circ}$, the $\Lambda$-shaped vortices become more distinctive and all of them are aligned both in the flow direction and the spanwise direction. Especially between $z=25$ and $z=35$ the heads of the $\Lambda$ shaped vortices start to move in the vertical direction and become more like hairpin vortices. At $\phi=162^{\circ}$, all the vortices become hairpin vortices in shape. Starting from $\phi=165^{\circ}$, the initially generated hairpin vortices populate the near-wall region, which can be seen from figure $4(f)$. This process continues until $\phi=180^{\circ}$ where the flow 
(a)

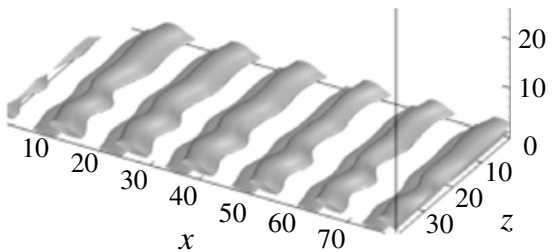

(c)

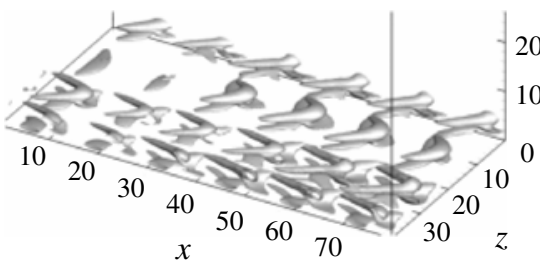

(e)

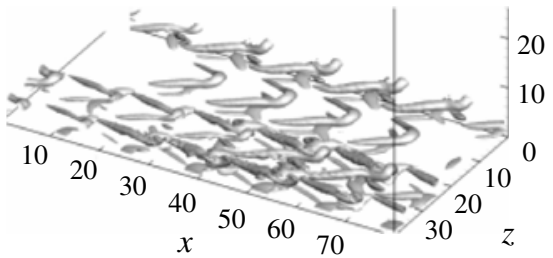

(g)

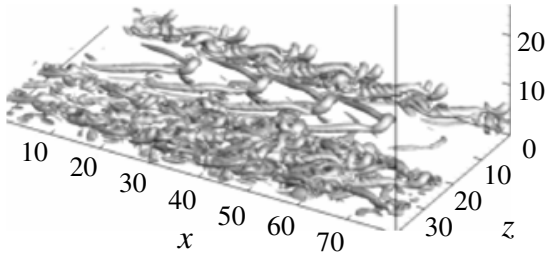

(b)

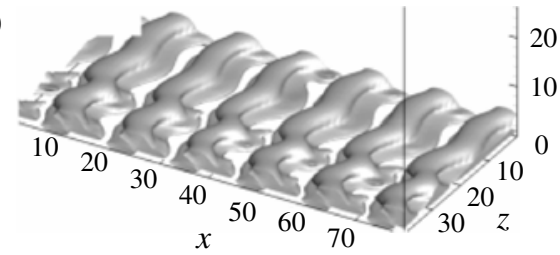

(d)

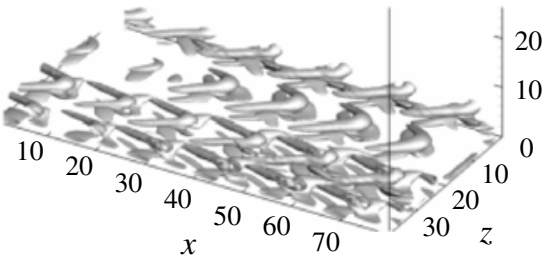

$(f)$

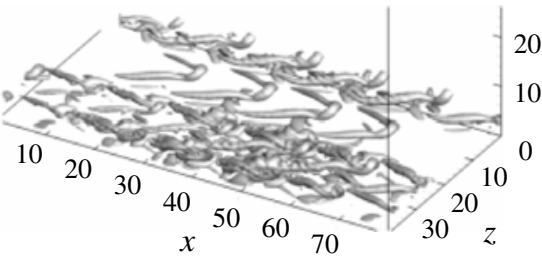

(h)

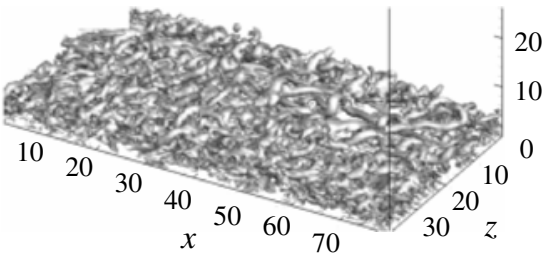

FIGURE 4. Iso-contours of $\lambda_{c i}^{2}$ for case R2000-0.20 at phases: $(a) \phi=114^{\circ} ;(b) \phi=144^{\circ}$; (c) $\phi=156^{\circ} ;(d) \phi=159^{\circ} ;(e) \phi=162^{\circ} ;(f) \phi=165^{\circ} ;(g) \phi=168^{\circ} ;(h) \phi=180^{\circ}$.

becomes comparatively chaotic (figure $4 h$ ). It should be pointed out that, after the flow shows transitional characteristics, the flow may become fully turbulent or just remain transitional, which we shall discuss in more detail in $\S 3$. We use the term 'chaotic' to refer to the flow state that shows transitional characteristics, but has not developed into a fully turbulent state.

Figure 5 shows the vortex structures at $R e_{\delta}=2500$ (case R2500-0.01). This case is selected as the generated vortices distinctly degenerate into turbulence towards the end of the wave period. At $\phi=72^{\circ}$ and $\phi=78^{\circ}$, we can clearly distinguish five pairs of rollers, almost equally spaced in the streamwise direction. In each pair, the one with smaller diameter is located closer to the wall and the other with larger diameter is located more towards the far stream. At $\phi=102^{\circ}$ (see figure $5 c$ ), the rollers are more energetic with higher $\lambda_{c i}$ values. Then at $\phi=108^{\circ}$ (figure $5 d$ ), the stretching in the roller pairs becomes more evident and the upper rollers are lifted up to $y=5 \sim 10$. At $\phi=114^{\circ}$, the upper rollers which were relatively straight in the spanwise direction at $\phi=108^{\circ}$ start deforming. At $\phi=117^{\circ}$, the initial formation of rib vortices around the rollers is observed. This suggests that the rollers become more energized to excite the surrounding flow to form additional vortex structures. Then, the aforementioned 

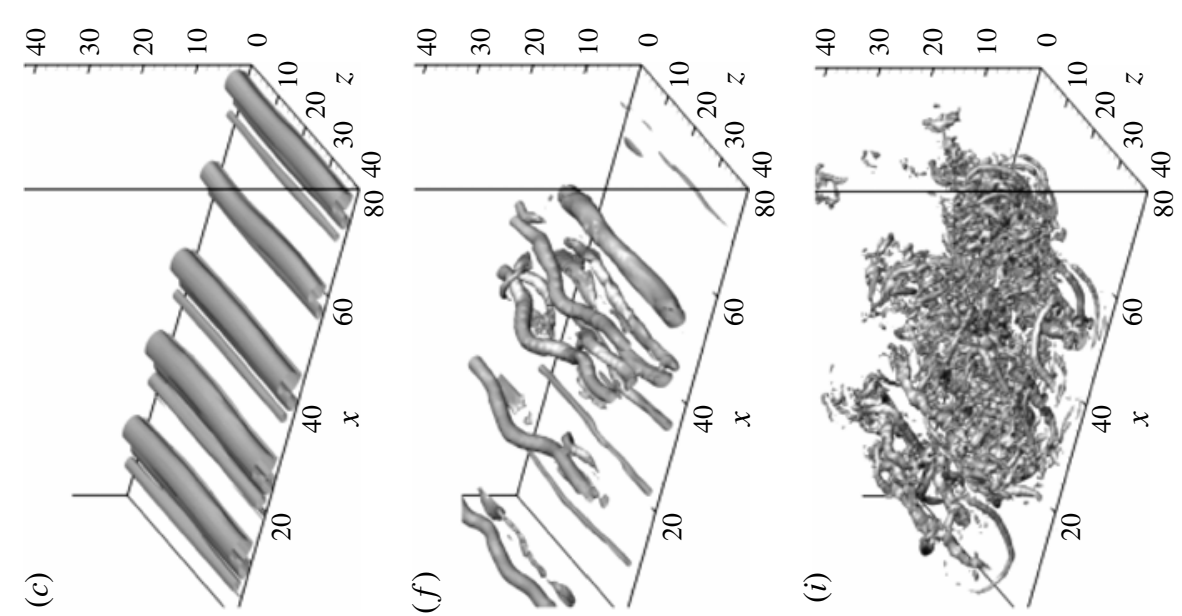

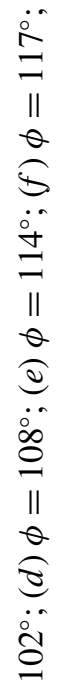
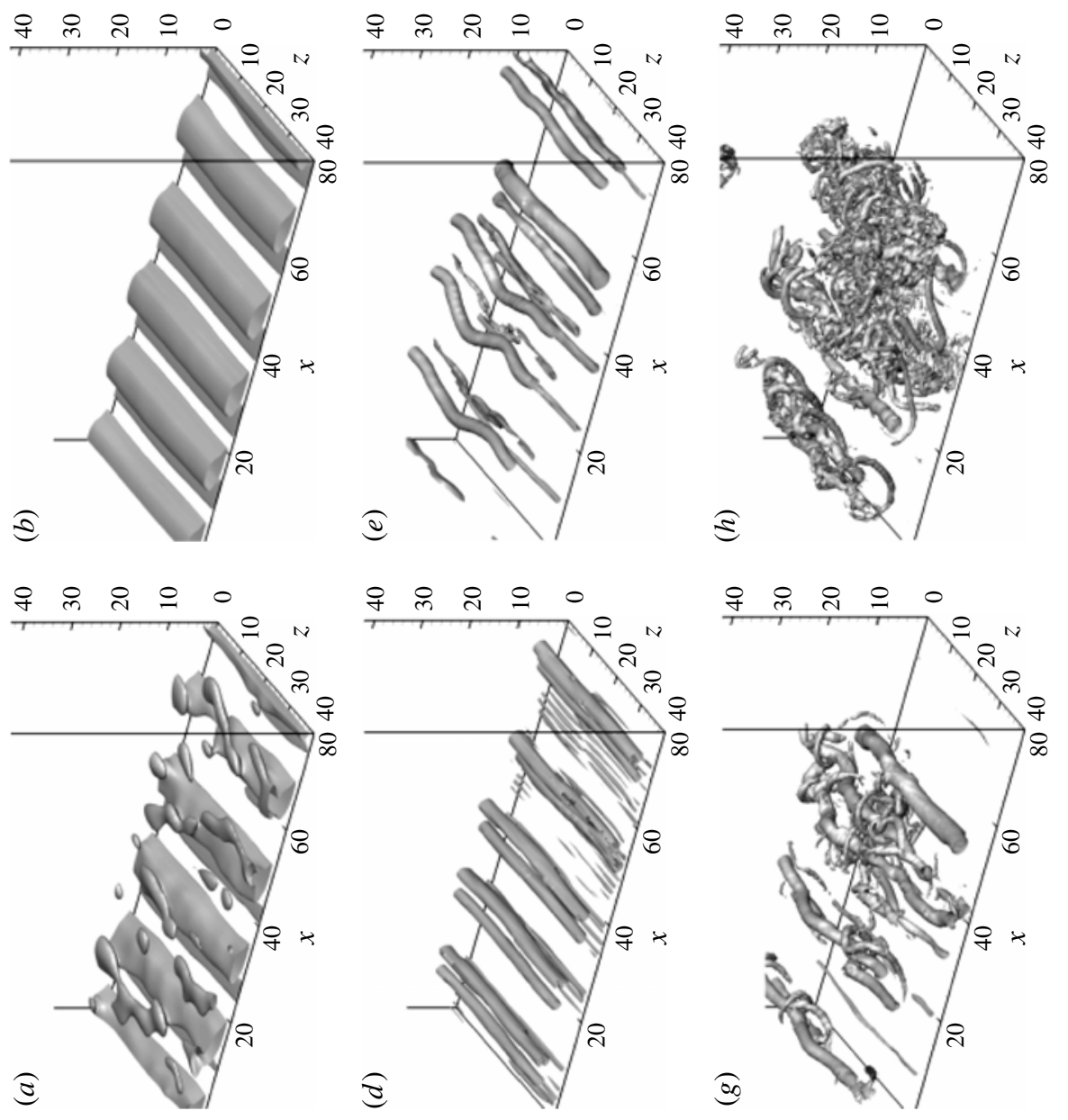

ชิำ 文 II

$11 \stackrel{2}{2}$

$\theta=$

ㅇํำ ำ 112 $\theta \equiv$ ㅎํㅇ $\ddot{\otimes} \frac{1}{11}$

을 $\approx \theta$

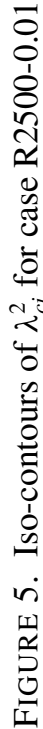


rib vortices become more populated. At $\phi=120^{\circ}$, we observe the initial degeneration of the rollers and the rib vortices into smaller chaotic structures. This degeneration becomes quite evident at $\phi=126^{\circ}$ and $\phi=132^{\circ}$ (figure $5 h, i$ ), which leads to a total transformation of the coherent vortices into chaotic motion in the remainder of the wave period (not shown).

At $R e_{\delta}=1500$, the generation of pairs of rollers near the bottom wall marks a distinct difference from the complete laminar condition. There are small-amplitude velocity fluctuations on top of the laminar velocity field as a result of the instability. As mentioned in Sumer et al. (2010), these fluctuations are observed in time series of point velocity measurements. However, these fluctuations vanish and the velocity profiles match the laminar solutions if averaged over a sufficient number of wave periods or plane-averaged at a fixed vertical point, which is the case in our study. At $\operatorname{Re}_{\delta}=2000$, the observations of the vortex structures illustrate the initiation of boundary layer instability. The rollers deform into $\Lambda$-shaped vortices and then deform into hairpin vortices. Based on this observation, we can conclude that at $\operatorname{Re}_{\delta}=2000$, the breakdown process of the spanwise rollers follows the laminar to turbulent transition sequence in a flat-plate boundary layer. On the other hand, at $R e_{\delta}=2500$, the rollers move in the vertical direction, become energized and are surrounded by rib vortices. Therefore, a different breakdown process is present for $\operatorname{Re}_{\delta}=2500$. The generation, evolution and fate of these vortex structures for the three cases presented here mark the main qualitative differences that distinguish these flow regimes. More quantitative discussions are presented in the next section.

\subsection{Mean and root-mean-square (r.m.s.) velocity profiles}

The interplay between the flow instability and mean velocity profile, together with the intensity and spatial characteristics of coherent structures and turbulence, can also be monitored through plane-averaged mean and r.m.s. velocity profiles. Also, the transformation from laminar to transitional and transitional to turbulent flow can be quantitatively diagnosed through these quantities. In this section, we present the vertical profiles of plane-averaged velocity and r.m.s. of velocity fluctuations for the same cases discussed in $\S 3.1$ and for an additional case of R4000-0.01. Figure 6 shows $(a)$ the plane-averaged velocity profiles and (b) r.m.s. velocity fluctuations for case R1500-0.20 (solid curves). For reference, the solution of laminar velocity profiles (Liu \& Orfilia 2004) is shown with symbols in figure 6(a). The velocity profiles closely agree with the laminar velocity profiles through the whole wave period. As discussed in $\S 3.1$ (see figure 3), although the instability takes place between $\phi=90^{\circ}$ and $\phi=180^{\circ}$, the generated fluctuations are not sufficient to alter the mean velocity profile. Another interesting observation is that at $\phi=90^{\circ}$, the velocity profile has the sharpest variation (largest vertical shear) below the maximum velocity. This is also the phase when the coherent vortices appear between $y=0$ and 1 . More detailed information on the growth of velocity fluctuations can be identified via planeaveraged r.m.s. velocity profiles (see figure $6 b$ ). At $\phi=-90^{\circ}, U_{r m s}$ values in the boundary layer, i.e. $y=[0,3]$, are smaller than those outside. Above the boundary layer, the fluctuations are reminiscent of the initial velocity fluctuations. As expected, these initially prescribed velocity fluctuations decay in time at later phases. Between $\phi=-60^{\circ}$ and $\phi=-30^{\circ}$, a slight increase in $U_{r m s}$ at $y=1$ can be observed in the form of a subtle peak. From $\phi=-60^{\circ}$ to $\phi=90^{\circ}$, the growth continues and after $\phi=90^{\circ}$, the peak value in $U_{r m s}$ decreases slightly (not shown). The layer where the growth in $U_{r m s}$ is observed extends in time and closely correlates with the boundary layer thickness (see also figure $6 a$ ). It is also noted here that the growth of velocity 

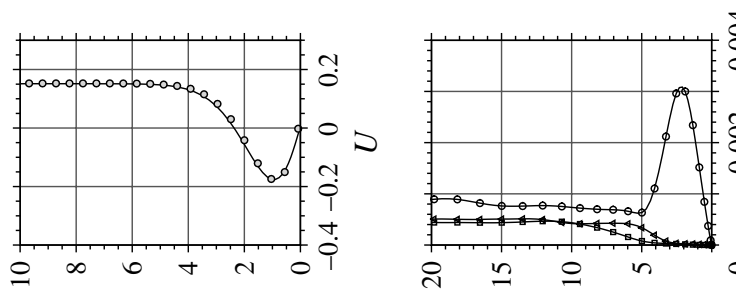

芯 き

के

$\stackrel{0}{\Xi}$

¿

迎?

क人

覀

กิ

$\therefore$

$\therefore \frac{2}{0}$
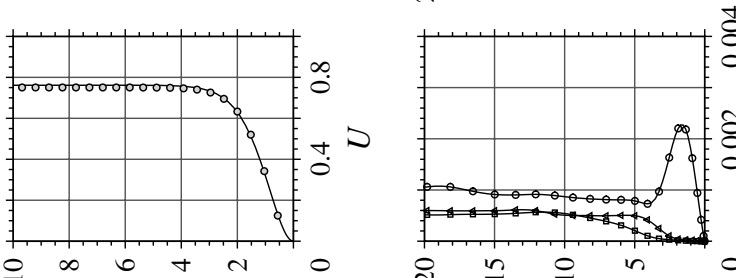

$\dot{\infty}$

ए

융

। ह

$\circ N$

웅
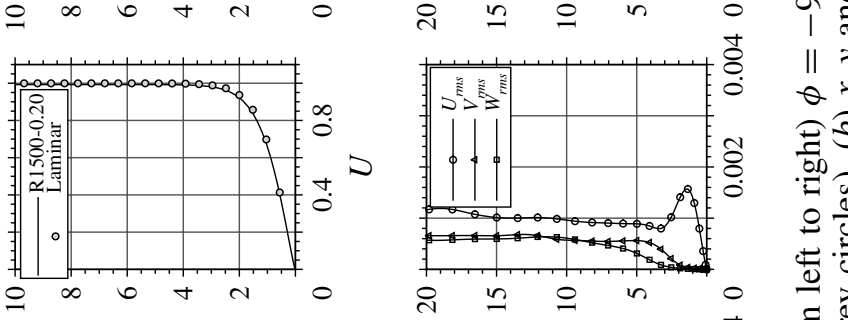

$\|>$

$\theta+5$

을

.0.0

$9 \frac{\pi}{5}$

过

छี
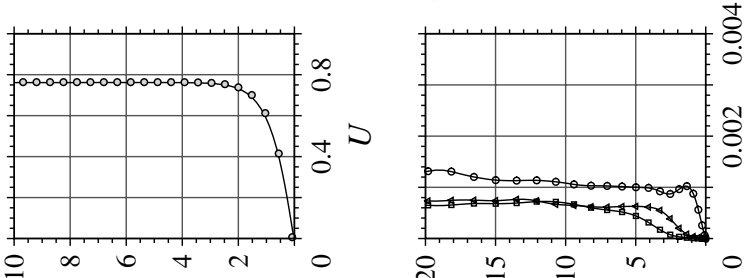

$\stackrel{8}{2}$

ㅎ

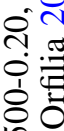
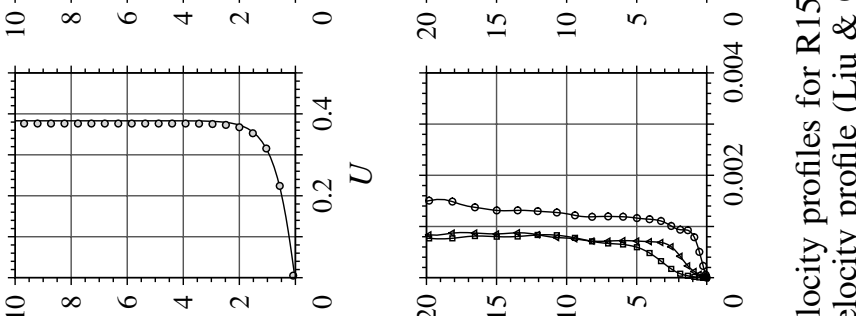

훌.己

\&

은

?.

:
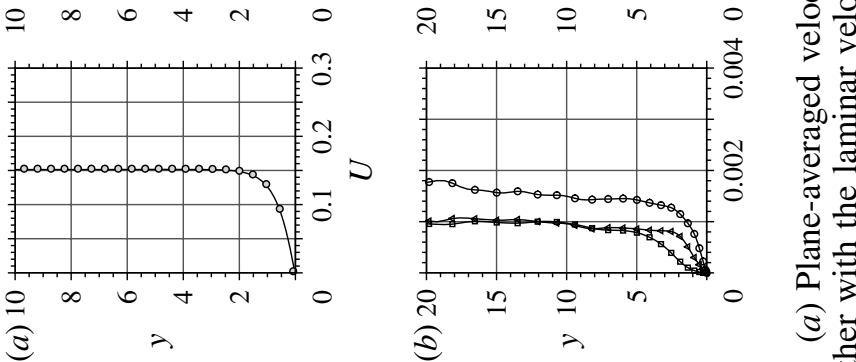

도 추

5 .

要

8

i

สิ

学

()

$6 \%$

디을

중

อี ฮี

可高 
fluctuations is almost completely in the streamwise direction, i.e. $U_{r m s}$, which is due to the rollers observed in figure 3 .

For case R2000-0.20, the plane-averaged velocity profiles match the laminar flow solution until between $\phi=150^{\circ}$ and $\phi=180^{\circ}$ (see figure $7 a$ ). At $\phi=180^{\circ}$, a noticeable deviation from the laminar solution can be clearly identified. According to figure $4(c)$, the phase of $\phi=156^{\circ}$ corresponds to the instant when the two-dimensional roller tubes transform into $\Lambda$-shaped vortices and it is the second stage (nonlinear stage) in the growth of the boundary layer instability, i.e. the secondary instability, following the roller pairs. At this second stage, the cascade of the energy from the leading wavelengths to higher wavelengths takes place through nonlinear growth. Therefore, the velocity profiles start to deviate from the laminar profile only after the initiation of the nonlinear growth. More insights can be revealed through r.m.s. of velocity fluctuations (figure $7 \mathrm{~b}$ ). From $\phi=0^{\circ}$ to $\phi=120^{\circ}$, the behaviour of the r.m.s. of velocity fluctuations is similar to that of $\mathrm{R} 1500-0.20$, i.e. the pronounced $U_{r m s}$ component due to the near-bottom rollers. At $\phi=120^{\circ}$, we observe a subtle increase in the $U_{r m s}$ profile between $y=0$ and 1 . This is when the vortex tubes closer to the wall start to energize. Then at $\phi=150^{\circ}$, this increase in $U_{r m s}$ becomes more pronounced and develops into a second extremum, that is located closer to the wall. In fact, this second extremum in $U_{r m s}$ overtakes the local peak located above it and becomes the stronger one. At this instant, we can also see a noticeable increase in $V_{r m s}$ and $W_{r m s}$ due to the $\Lambda$-shaped vortices (see also figure $4 c$ ). At $\phi=180^{\circ}$, all three components of velocity fluctuations become larger $\left(U_{r m s}\right.$ remains the largest) and extend further in the vertical direction up to $y=9 \sim 10$. Only after the nonlinear growth, which eventually leads to hairpin vortices, do we start to observe the development of velocity fluctuations in all three directions and a deviation of the plane-averaged velocity profile from the laminar solution.

Similarly, the first deviation from the laminar profile in case R2500-0.01 takes place between phases $\phi=90^{\circ}$ and $\phi=120^{\circ}$ where the roller pairs start to stretch and initial formation of the rib vortices occurs (figure $8 a$ ). The first noticeable deviation in the plane-averaged velocity profile from its laminar counterpart starts at $\phi=102^{\circ}$ (not shown) where the spanwise rollers are stretched and energized (see figure $5 c$ ). From the profiles of the r.m.s. of velocity components (figure $8 b$ ), it can be observed that the secondary peak of $U_{r m s}$ forms between phases $\phi=60^{\circ}$ and $\phi=90^{\circ}$. At $\phi=120^{\circ}$, velocity fluctuations in the spanwise direction $W_{r m s}$ are sufficiently developed and their magnitude is comparable to that of $U_{r m s}$. At $\phi=150^{\circ}$ all three components of velocity fluctuations are of similar magnitude, suggesting the development of more mature turbulence. Compared to R2000-0.20 the extent of high-velocity fluctuations in $\mathrm{R} 2500-0.01$ is more pronounced and can be observed up to $y=40$. The development into a more three-dimensional chaotic flow towards the end of the wave period is quite different from that observed in the previous two cases. More detailed discussion on this issue will be given in $\S 4.2$ through the power spectrum of velocity fluctuations and turbulent kinetic energy budget.

For R4000-0.01, the deviation from the laminar velocity profile starts even earlier, falling within the phases $\phi=60^{\circ}$ and $\phi=90^{\circ}$ (figure $9 a$ ). Similarly, in the r.m.s. velocity fluctuations (figure $9 b$ ), $U_{r m s}$ starts to grow at around $\phi=-30^{\circ}$, and the secondary peak in $U_{r m s}$ occurs between $\phi=30^{\circ}$ and $\phi=60^{\circ}$. Starting at $\phi=90^{\circ}$, the growth can be seen in all the velocity components due to the chaotic nature of the vortical structures as a result of nonlinear growth of the velocity fluctuations, which continues till the end of the wave period. 

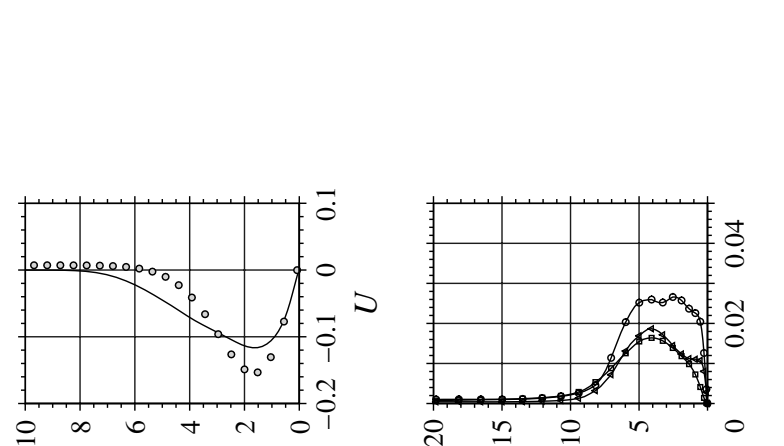

$\stackrel{\square}{\sharp}$

के

$\Xi$

궁

象

응

चे

유롤
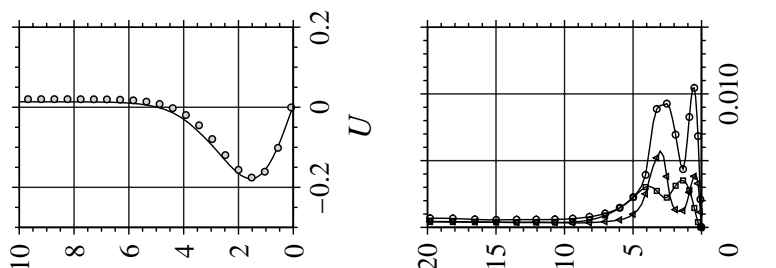

$\rightarrow-4$

$\dot{\mathrm{i}}$

긍

유융

司

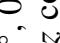
능ำ
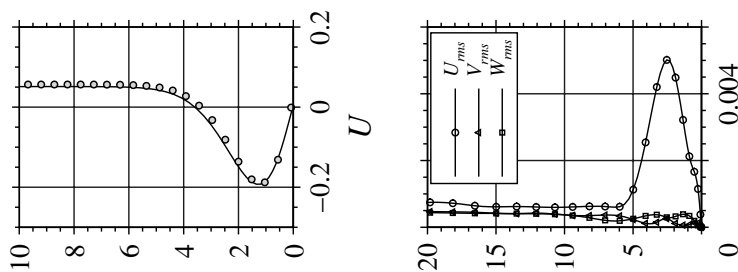

$11>$

$\theta+5$

Еิ

立

은

는

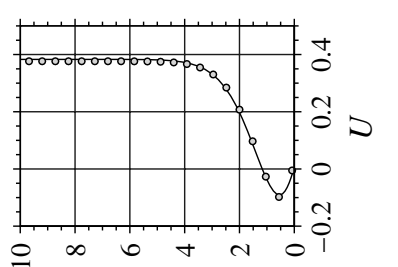

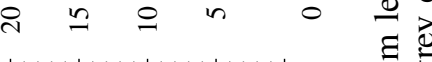
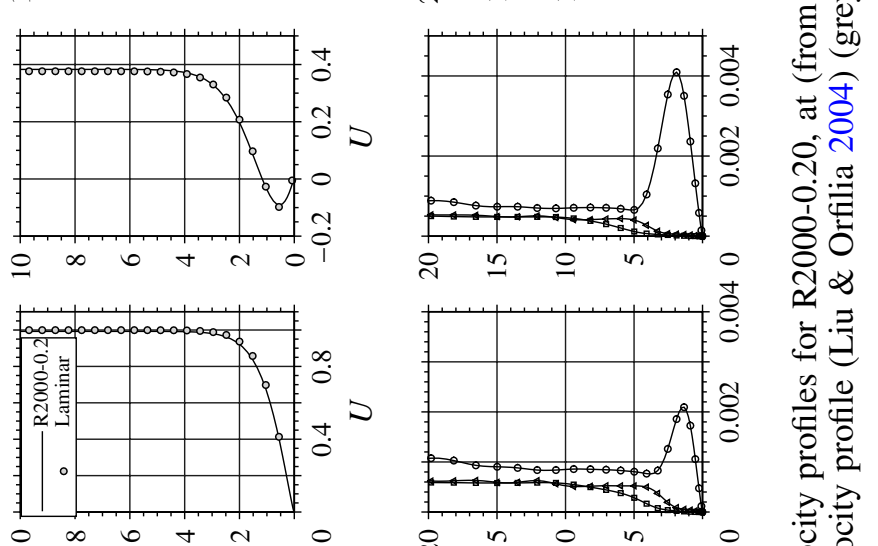

प तै

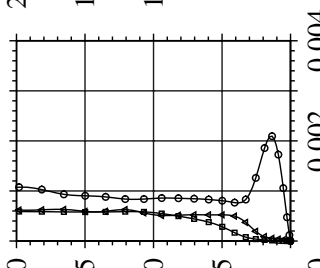

๖ี.

递递

긍

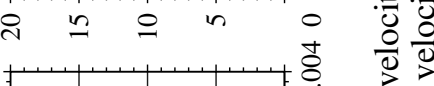

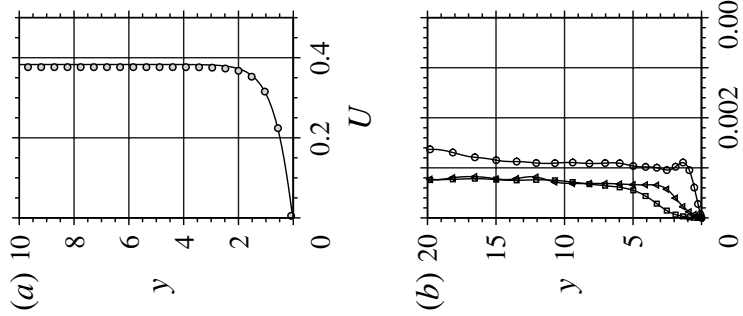

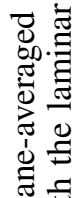

离

Q

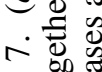

됭 을

৩

近. 施 

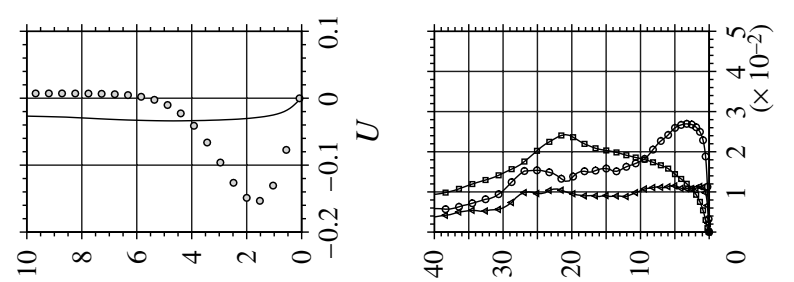

$\stackrel{\Xi}{\Xi}$
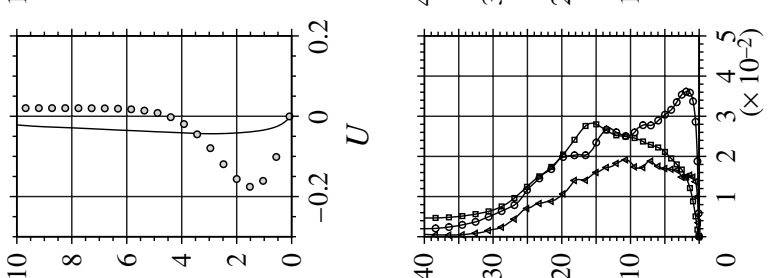

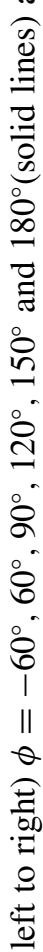
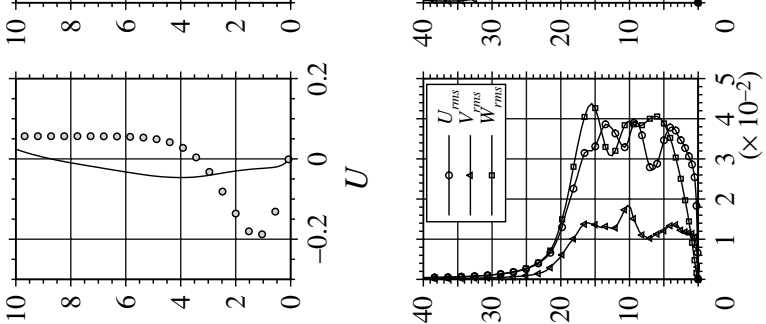

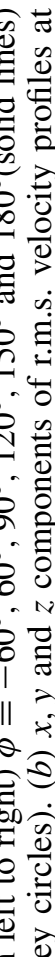
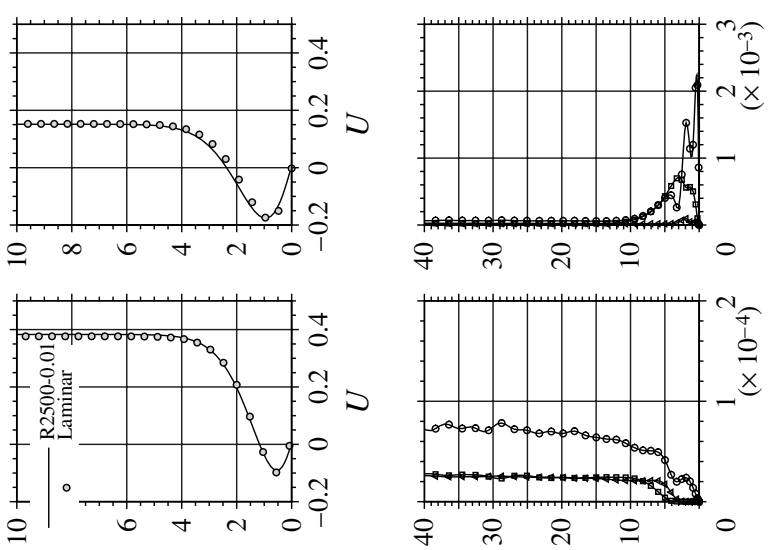

ठี

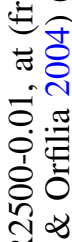

$\stackrel{\sim}{\approx} \equiv$

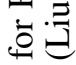

운

言兘

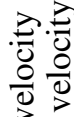
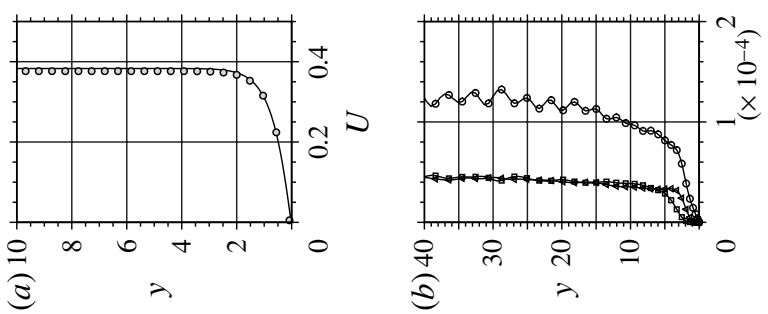

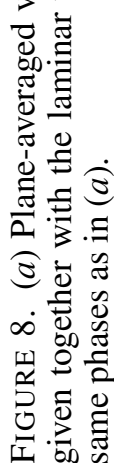


In all the cases presented in this section, the initiation of the growth in $U_{r m s}$ is at around $\phi=-60^{\circ}$ to $\phi=-30^{\circ}$. The timing of this initial growth in $U_{r m s}$ does not correlate with the Reynolds number but more with the initial amplitude of the velocity fluctuations. We shall discuss this issue in detail in $\S 4$. However, the secondary growth behaves quite differently from the initial growth. The timing of the occurrence of the second near-bottom maximum in $U_{r m s}$ marks the instant when the strength of the roller pairs becomes significant and the initiation of nonlinear growth. This secondary peak formation in the $U_{r m s}$ profile takes place at earlier phases with increasing Reynolds number and is nearly insensitive to the initial perturbation amplitude. Another observation is the near absence of vertical and spanwise components of the velocity fluctuations in the boundary layer during the initial growth in $U_{r m s}$. The vertical and spanwise velocity fluctuations start to increase and become significant only when the secondary peak in $U_{r m s}$ appears. This corresponds to the phase when the rollers deform into three-dimensional structures in the form of $\Lambda$-shaped vortices or rib vortices and the increase in $V_{r m s}$ and $W_{r m s}$ becomes significant. Furthermore, when the flow becomes more chaotic, the magnitudes of $V_{r m s}$ and $W_{r m s}$ become comparable to that of $U_{r m s}$.

\section{Discussion}

\subsection{Instability mechanisms}

In $\S 3.1$, we observed that formation of the spanwise rollers occurs at the deceleration phase of the solitary wave. The decelerating phase provides the generic conditions for inviscid instability as it gives rise to the formation of inflection point(s) due to adverse pressure gradient. On the other hand, at $R e_{\delta}=2000$, we also observed that transition from the laminar state to chaotic motion follows a path of secondary instability similar to the $K$-type secondary instability of a boundary layer. In this section, we shall provide more details on the instability mechanisms.

It was observed in the $U_{r m s}$ profiles presented in $\S 3.2$ that towards the mid to late stage of the acceleration phase, there is growth in $U_{r m s}$ close to the bottom boundary in the form of a local peak. To further investigate its origin, a time series of peak value in $U_{r m s}$ profiles, $U_{r m s}^{p}$, is given in figure 10 for the different $\varepsilon$ values at $R e_{\delta}=400,1500,2000$ and 2500. Recall that unlike $R e_{\delta}=2000$ and 2500, at $R e_{\delta}=1500$ there is no chaotic motion resulting from a breakdown of the twodimensional spanwise rollers. On the other hand, for $R e_{\delta}=2000$ and 2500, nonlinear growth occurs following the initial development of the spanwise rollers. Initially, $U_{r m s}^{p}$ occurs at a random location within the computational domain dictated by the prescribed initial random disturbance as it decays before the onset of initial growth. At $R e_{\delta}=400$, where the flow is laminar, $U_{r m s}^{p}$ (and the entire $U_{r m s}$ profiles) decays throughout the whole wave period regardless of the prescribed disturbance amplitude (see figure $10 a$ ). At higher $R e_{\delta}$, starting from $R e_{\delta}=500$, there is a growth in velocity fluctuations which can be seen in figure $10(b-d)$. The period between the start of the wave $\left(\phi=-180^{\circ}\right)$ and the onset of observed growth is longer for smaller $\operatorname{Re}_{\delta}$ and $\varepsilon$. The increase in $U_{r m s}^{p}$ following the initial decay is rather abrupt. In other words, the time rate of change in $U_{r m s}^{p}$ just before and after the initiation of the aforementioned growth is different in magnitude. This can be elucidated in more detail. In $\S 3.2$, we have observed the growth in $U_{r m s}$ within the near-wall layer to be in the form of a local extremum. Therefore, the peak value of $U_{r m s}$ within the boundary layer can easily be traced as long as this extremum exists, and is shown in figure 10 with open circles. Initially, the maximum $U_{r m s}$ value within the boundary layer is smaller than 

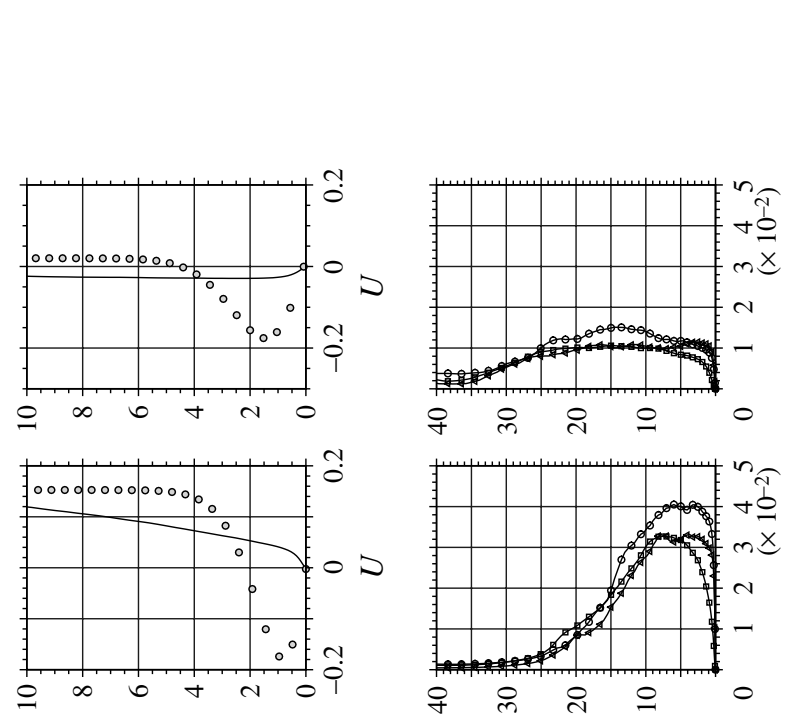

$\stackrel{\mathscr{E}}{\Xi}$

○

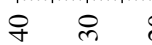

के

$\stackrel{\Xi}{\Xi}$

응

$\stackrel{\oplus}{\oplus}$

는

ซ్ర

○ छ

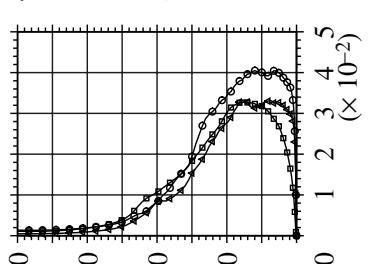

a.

$\stackrel{0}{0}$

苛

$\dot{\circ}$

m

๑ छี

$\stackrel{8}{\circ}$

\& $\stackrel{2}{1} 0$ |
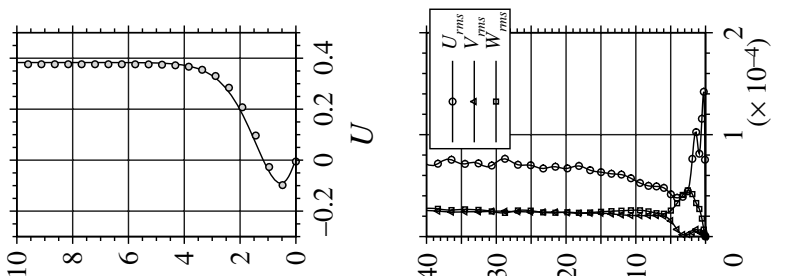

$\theta=$

동

$\exists$

용

$\stackrel{\varpi}{0}$

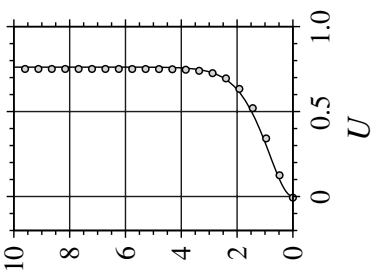

ㅇ
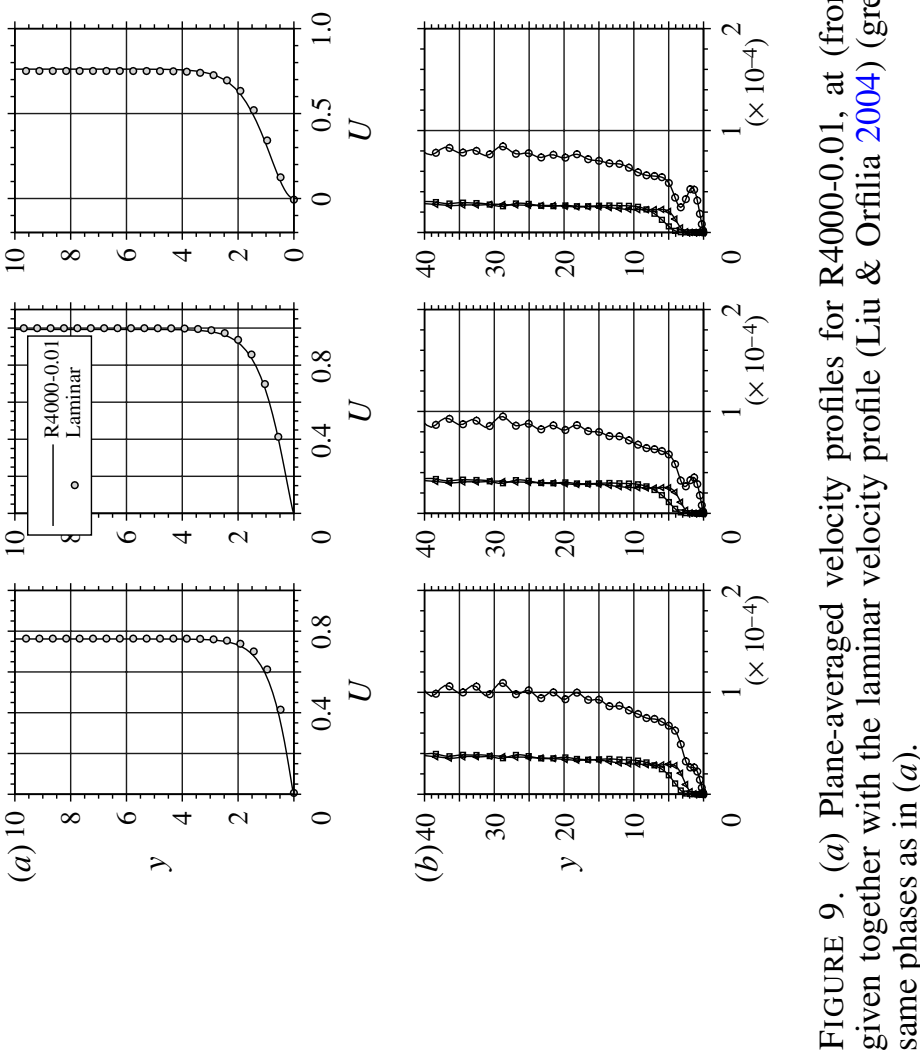

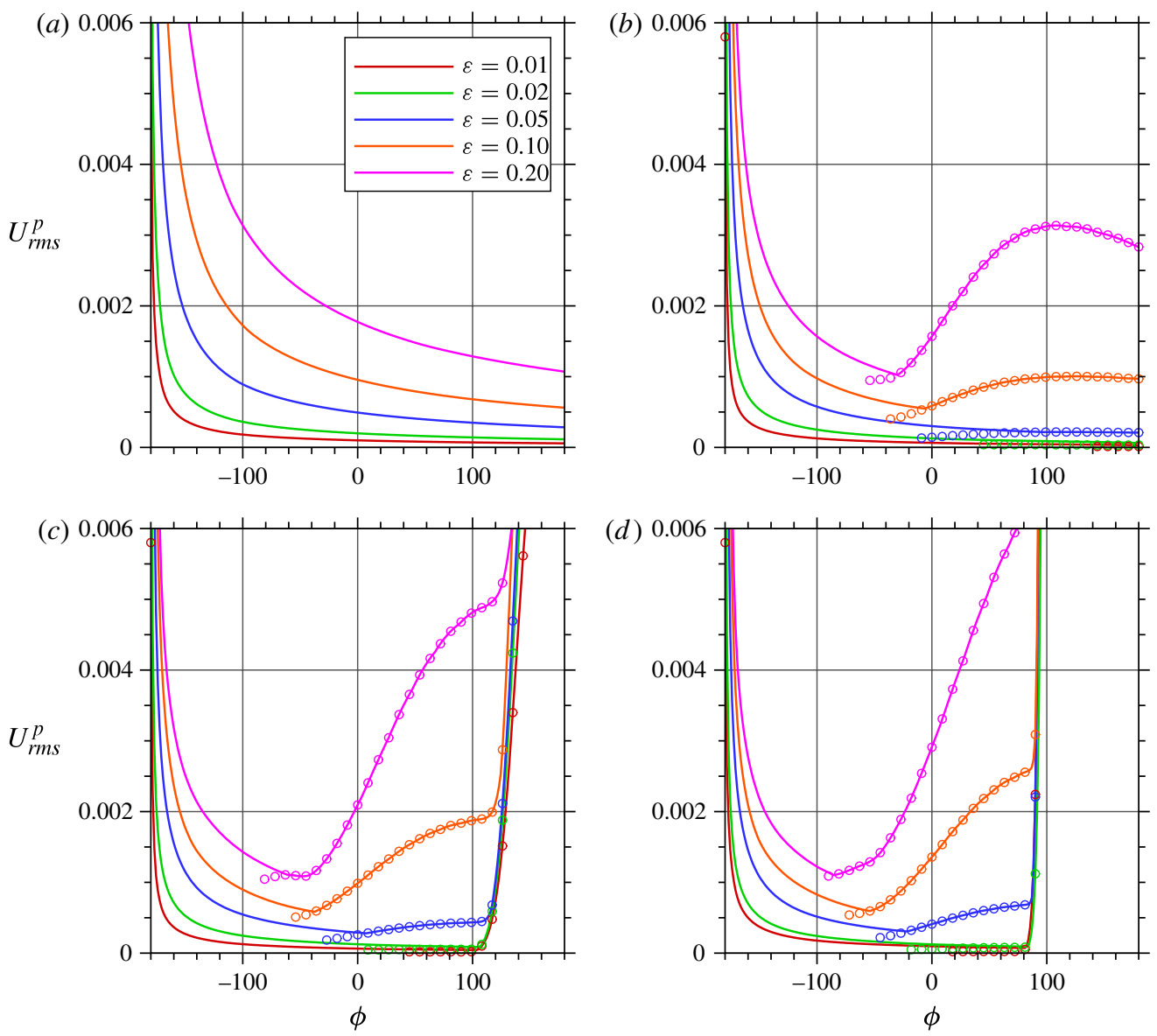

FIGURE 10. Time series of maximum $U_{r m s}, U_{r m s}^{p}$, in a wave period for all the levels of initial fluctuating velocity amplitude for: $(a) R e_{\delta}=400 ;(b) R e_{\delta}=1500 ;(c) R e_{\delta}=2000$; and (d) $R e_{\delta}=2500$. Solid lines show $U_{r m s}^{p}$ in the whole computational domain and the open circles show $U_{r m s}^{p}$ in the boundary layer.

that outside. Outside the boundary layer, the fluctuations continue to decay, whereas if favourable conditions are present, the fluctuations grow inside the boundary layer. At a certain phase, the fluctuations inside the boundary layer outgrow the outside fluctuations and $U_{r m s}^{p}$ is seen to increase in time.

The nonlinear nature of the disturbance evolution can be observed at all the Reynolds numbers considered. At $\operatorname{Re}_{\delta}=1500$, even though the disturbance continued to decay, the plots of $U_{r m s}^{p}$ in figure $10(b)$ cannot be collapsed when scaled with $\varepsilon$. As shown in figure 11 a plot of $U_{r m s}^{p}$ versus $\varepsilon$ at $\phi=100^{\circ}$ shows that the scaling is linear only for small values of initial disturbance amplitude. With increasing amplitude of initial disturbance, the decay seems slower, as indicated by the larger value of $U_{r m s}^{p}$ than would be predicted by linear scaling.

At higher Reynolds numbers the role of nonlinearity is even clearer. From the time series of $U_{r m s}^{p}$ it can be seen that there exists a viscous instability during the acceleration phase. The viscous nature of the instability can be conjectured from the lack of inflection point in the mean velocity profile during the acceleration phase. 

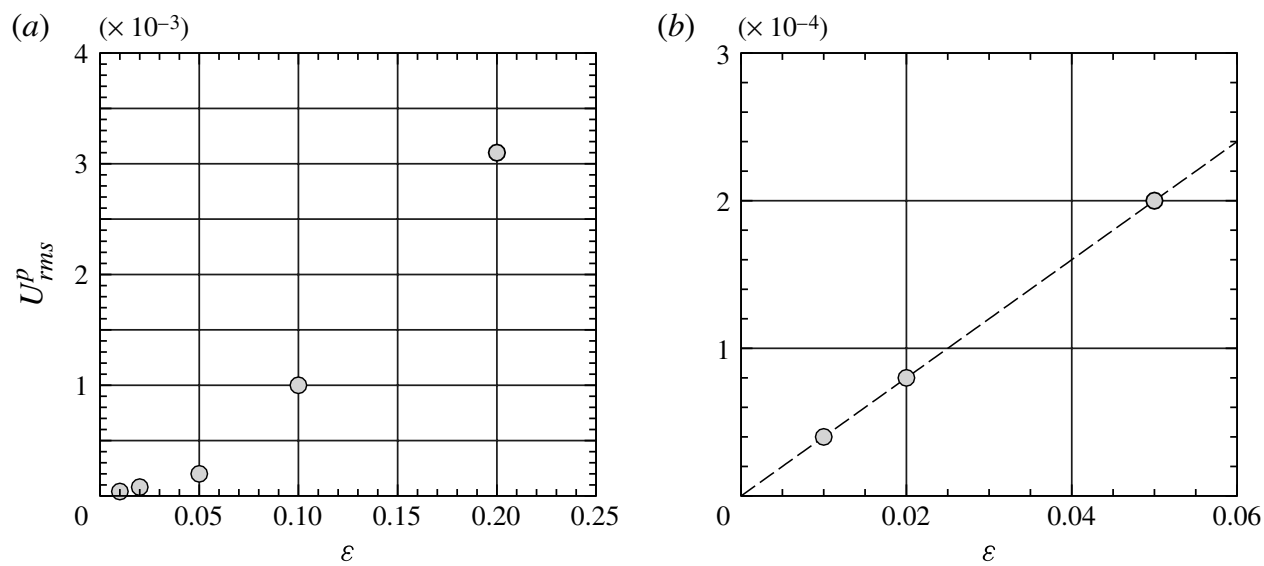

FIGURE 11. (a) Maximum of $U_{r m s}^{p}$ observed at $\phi=100^{\circ}$ for all values of initial amplitude of velocity fluctuations, $\varepsilon$, at $\operatorname{Re}_{\delta}=1500$. (b) Magnified version of $(a)$ with only $\varepsilon=0.01,0.02$ and 0.05 . While there is a perfect linear variation in $(b)$, deviation from linear variation is observed at $\varepsilon=0.1$ and 0.2 in $(a)$.

The formation and the growth of this instability depend not only on $\operatorname{Re}_{\delta}$ but also on the initial disturbance amplitude. The dependence on the amplitude of the initial background disturbance suggests that the instability is likely to be nonlinear. However, this instability does not lead to significant growth in velocity fluctuations and hence breakdown to turbulence. What seems to lead to turbulence, depending on $\operatorname{Re}_{\delta}$, is the stronger instability that occurs during the deceleration phase due to the adverse pressure gradient.

To further examine the instabilities, we computed the Fourier transform of the fluctuating streamwise velocity along the streamwise direction. The mean-square fluctuating streamwise velocity, $\left\langle U^{\prime} U^{\prime}\right\rangle$, was computed by averaging over the spanwise direction. Contours of the logarithm of this quantity for $R e_{\delta}=1500$ are plotted in figure 12 as a function of phase and distance from the bottom boundary. The results for the first seven streamwise Fourier modes $\left(\kappa_{x}=1, \ldots, 7\right)$ are presented for three different amplitudes of initial disturbance. As can be seen, in all seven wavelengths, the decay rate close to the bottom wall is larger than those towards the free stream during the acceleration phase. There is an instantaneous growth in $E_{\left\langle U^{\prime} U^{\prime}\right\rangle}$ for $\kappa_{x}=1$ within $y=[0.5,2]$ at around $\phi=\left[-100^{\circ},-80^{\circ}\right]$ which is observed only for $\varepsilon=0.2$ (see figure 12ci). In the same simulation, the rest of the wavelengths continue to decay over the entire accelerating phase. It should be noted that for $\varepsilon=0.2$ the vertical location and the instant when the growth in $E_{\left\langle U^{\prime} U^{\prime}\right\rangle}$ starts correspond to where and when the maximum $U_{r m s}$ is first observed. After $\phi=-80^{\circ}$ the growth close to the bottom wall is followed by decay. But the local disturbance growth due to the instability migrates rapidly in the vertical direction. But by the end of the acceleration phase this instability has completely vanished. While the growth is only observed for $\varepsilon=0.2$ at $R e_{\delta}=1500$, it can be observed that the decay rate for $\varepsilon=0.1$ is smaller compared to those of $\varepsilon=0.01,0.02$, and 0.05 .

For $R e_{\delta}=2500$, similar observations to those for $R e_{\delta}=1500$ can be made. The only difference is that the growth during the acceleration phase is clearly observed for both $\varepsilon=0.1$ and 0.2 (see figure 13). Similar to $R e_{\delta}=1500$, the growth is short-lived and migrates quickly towards the free stream. Again the vertical location and the phase at 
(ai)

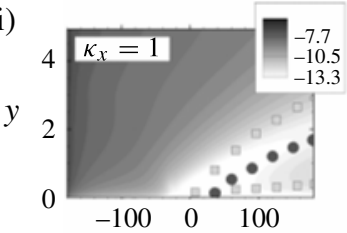

(aii)

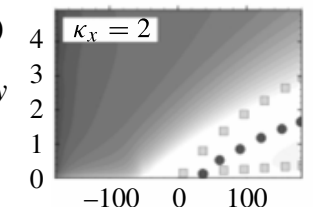

(aiii)

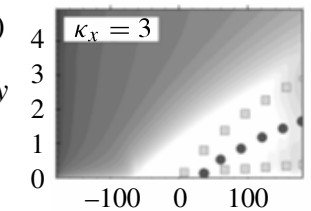

(aiv)
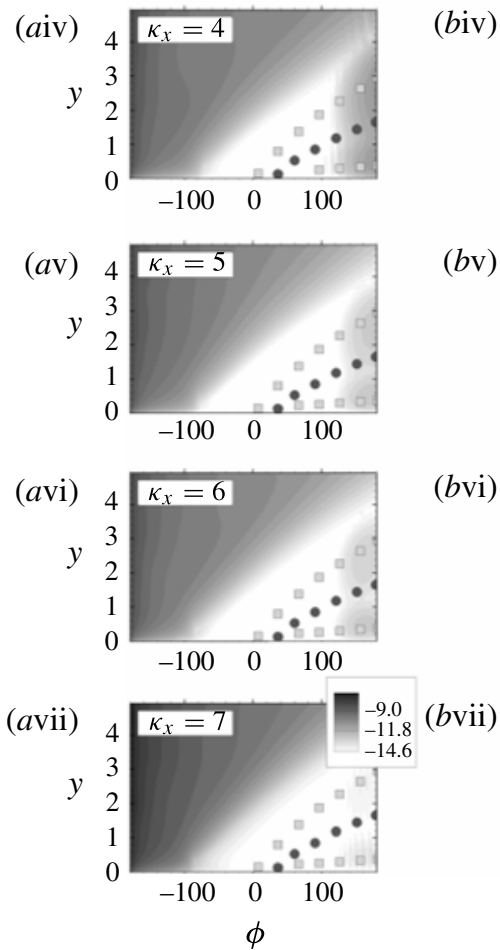

(bi)

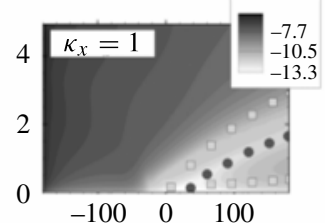

(bii)

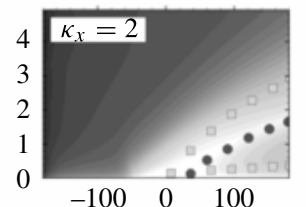

(biii)

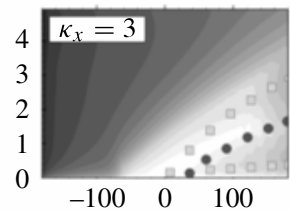

(biv)

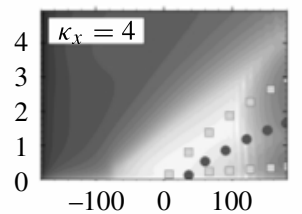

(bv)

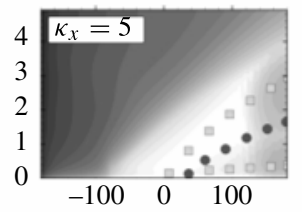

(bvi)

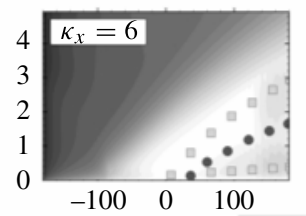

(ci)

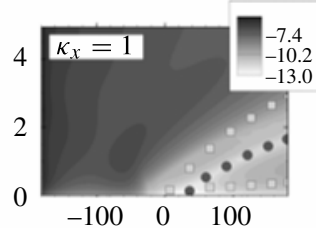

(cii)

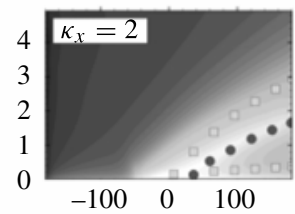

(ciii)

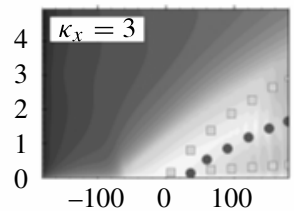

(civ)

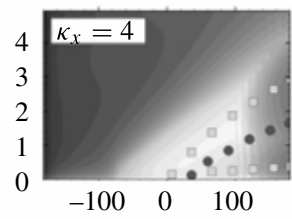

(cv) ${ }_{4} \kappa_{x}=5$

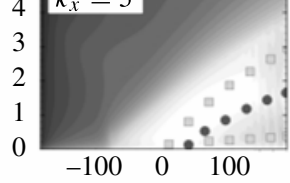

(cvi) ${ }_{4} \kappa_{x}=6$
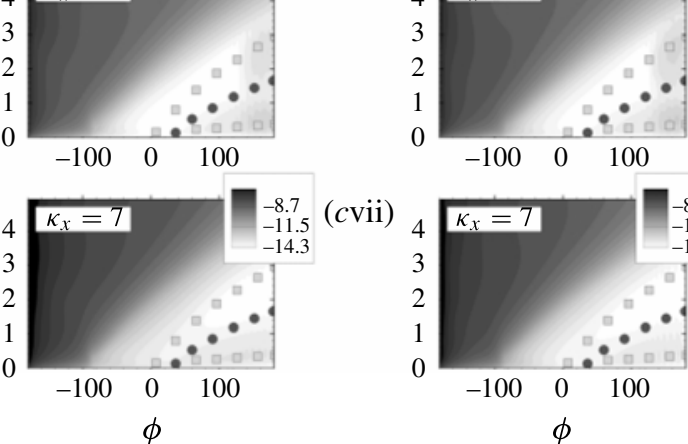

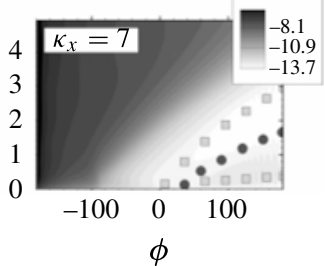

FIGURE 12. Contours of streamwise velocity fluctuation energy in logarithmic scale, $\log \left(E_{\left\langle U^{\prime} U^{\prime}\right\rangle}\right)$, at various wavelengths at $R e_{\delta}=1500$. Columns $(a),(b)$ and $(c)$ show $\log \left(E_{\left\langle U^{\prime} U^{\prime}\right\rangle}\right)$ of simulations R1500-0.02, R1500-0.1, and R1500-0.2, respectively. Rows i to vii correspond to the first seven wavelengths of the spectra, $\kappa_{x}=1$ to 7 , respectively. Dark grey circles correspond to the locations where mean velocity extrema are observed. Light grey squares correspond to the inflection points. 
which the growth of the $\kappa_{x}=1$ streamwise mode is observed closely match those of $\mathrm{R} 1500-0.2$. Therefore, based on these results the viscous instability can be concluded to be a long-wave nonlinear instability.

In figure 12 for $\operatorname{Re}_{\delta}=1500$ during the decelerating phase, comparatively low energy in $E_{\left\langle U^{\prime} U^{\prime}\right\rangle}$ can be observed close to the vertical location where the mean streamwise velocity reaches an extremum. The time evolution of these extremum points is marked as dark grey circles in figure 12. Both above and below the extremum there is increase in $E_{\left\langle U^{\prime} U^{\prime}\right\rangle}$ for all wavelengths shown. Also shown in figure 12 are the locations of the inflection points. The first inflection point that is located above the streamwise velocity extremum starts at around $\phi=0^{\circ}$. Later a second inflection point also appears in the mean streamwise velocity profile, which stays very close to the bottom boundary. Both these sets of inflection points are marked as light grey squares in figure 12 .

For all the streamwise wavelengths, the maximum of the energy is well correlated with the location of the inflection points. The increase in $E_{\left\langle U^{\prime} U^{\prime}\right\rangle}$ is the strongest for $\kappa_{x}=4$, which in turn corresponds to the shortest distance between two consecutive rollers that we observe in figure 3. In the case of $R e_{\delta}=2500$, there is a strong secondary instability and the nonlinear growth in the fluctuations after $\phi=100^{\circ}$ has already been presented in $\$ 3$. In figure 13, this can be observed from the high energy level of $E_{\left\langle U^{\prime} U^{\prime}\right\rangle}$ just after $\phi=100^{\circ}$. Between $\phi=0^{\circ}$ and $\phi=100^{\circ}$, the growth pattern is quite similar to the one for R1500-0.2 as the minimum of $E_{\left\langle U^{\prime} U^{\prime}\right\rangle}$ is around the extremum of the mean streamwise velocity and the peak growth in $E_{\left\langle U^{\prime} U^{\prime}\right\rangle}$ is well correlated with the inflection points. At $\operatorname{Re}_{\delta}=2500$ it is not very clear which streamwise mode has the highest energy as the fluctuations grow rapidly and become nonlinear. However, there are close similarities in the variation of the energy of velocity fluctuations among all the wavelengths. Initially, the level of $E_{\left\langle U^{\prime} U^{\prime}\right\rangle}$ ranges between $10^{-8}$ and $10^{-10}$, which is quite low. During this early deceleration phase, since the coherent vortex structures are nearly two-dimensional with no sign of nonlinearity, we conclude that the instability observed before breakdown during the deceleration phase is linear. Since the growth of $E_{\left\langle U^{\prime} U^{\prime}\right\rangle}$ is observed in several different streamwise modes, this linear instability can be considered to be a broadband instability.

The initiation of the instability during the deceleration phase is at about $\phi=35^{\circ}$, which is when the mean velocity extremum and second inflection point, which is closer to the wall, first emerge. It should be noted that the observed growth in velocity fluctuations takes place earlier in the deceleration phase and not immediately following the initial formation of the two-dimensional rollers. It is very likely that the velocity fluctuations at the initial stage of the linear instability are too small to be visualized as rollers, which was also mentioned by Blondeaux et al. (2012). Only significant growth in velocity fluctuations would lead to noticeable two-dimensional vortex structures. The critical phase when the linear instability is initiated is estimated to be closer to those found in the theoretical study of Blondeaux et al. (2012). The growth rate of the disturbance after its initiation is dependent on $R e_{\delta}$, and if $R e_{\delta}$ is sufficiently high, the fluctuations grow rapidly and become nonlinear. Therefore, breakdown of the two-dimensional rollers and the onset of turbulence discussed in $\S 3$ can be interpreted to be the result of instability during the deceleration phase.

\subsection{Nonlinear growth and turbulence}

The different pathways of transition of two-dimensional rollers into a chaotic state and possibly turbulence also shows variation as was observed in $\S 3.1$. At $R e_{\delta}=2000$ the rollers are distorted into $\Lambda$-shaped vortices which are all aligned (see figure 4 ). 
(ai)

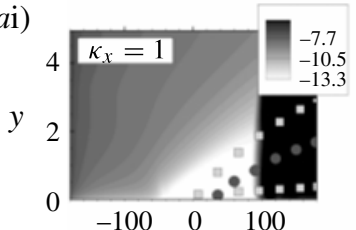

(aii)

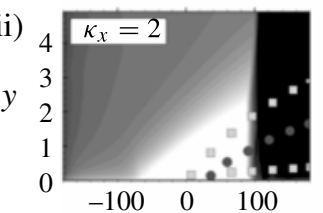

(aiii)
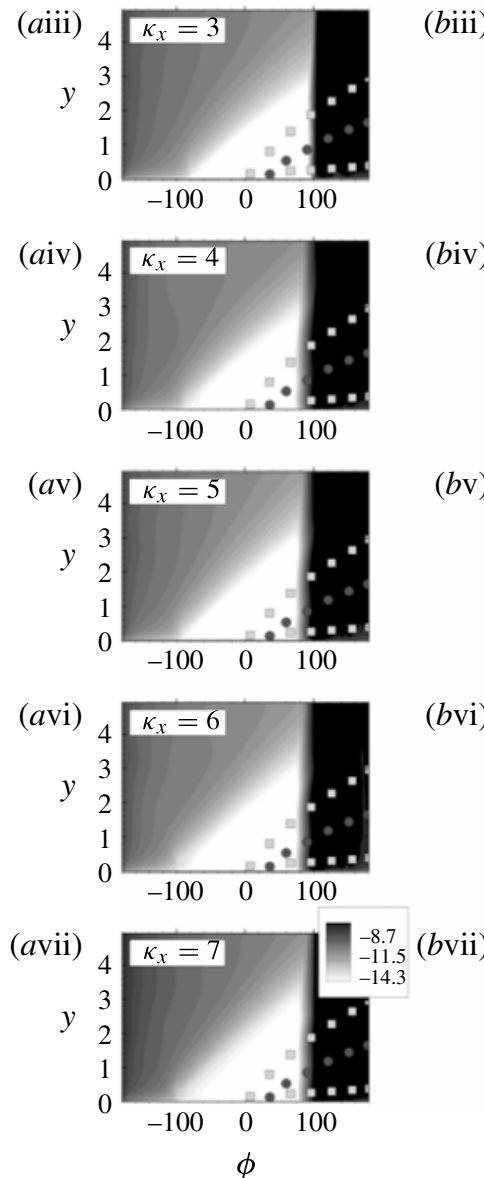

(bi)

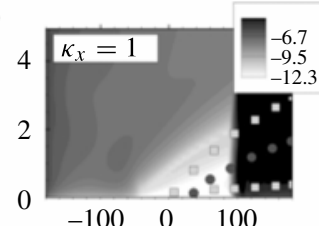

(bii)

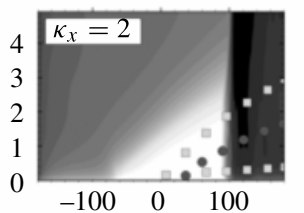

(biii)

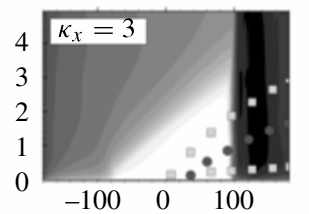

(biv)

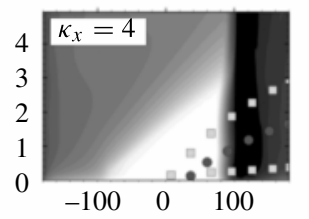

(bv)

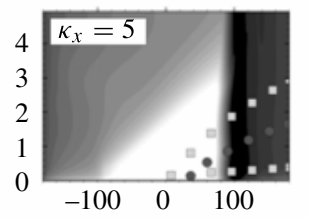

(bvi)

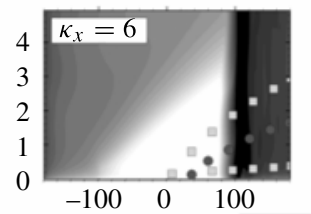

(ci)

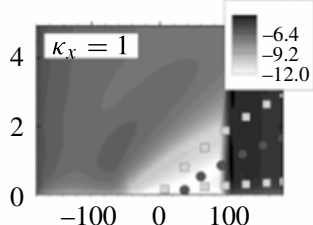

(cii)

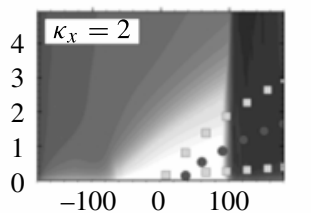

(ciii)

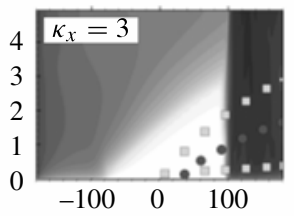

(civ)

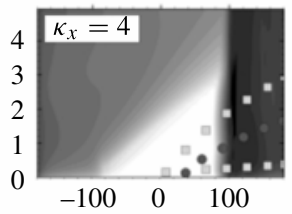

$(c \mathrm{v})$

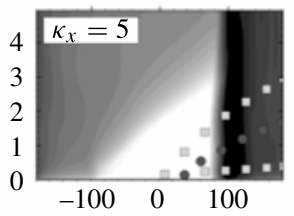

(cvi)

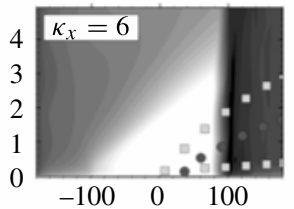

FIGURE 13. Contours of streamwise velocity fluctuation energy in logarithmic scale, $\log \left(E_{\left\langle U^{\prime} U^{\prime}\right\rangle}\right)$, at various wavelengths at $\operatorname{Re}_{\delta}=2500$. Columns $(a),(b)$ and $(c)$ show $\log \left(E_{\left\langle U^{\prime} U^{\prime}\right\rangle}\right)$ of simulations R2500-0.02, R2500-0.1, and R2500-0.2, respectively. Rows i to vii correspond to the first seven wavelengths of the spectra, $\kappa_{x}=1$ to 7 respectively. Dark grey circles correspond to the locations where mean velocity extrema are observed. Light grey squares correspond to the inflection points. 


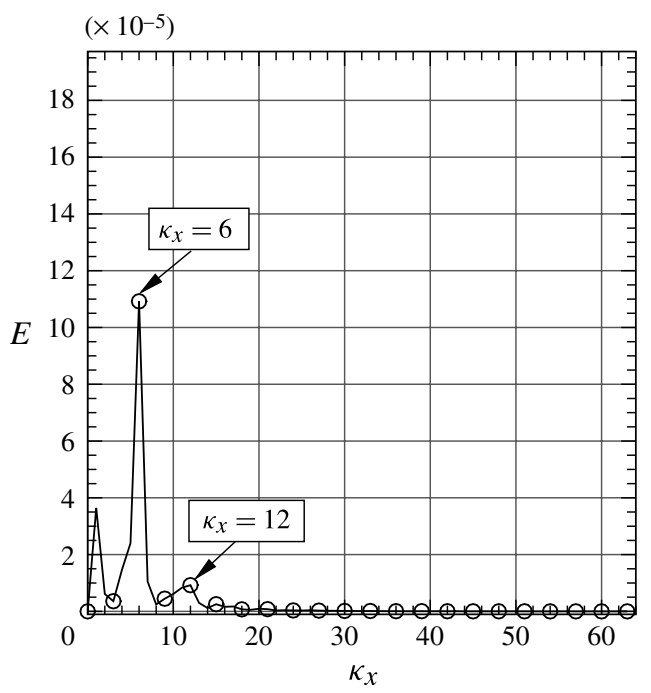

FIGURE 14. Power spectra of the streamwise velocity fluctuations at $y=1.0$ for case R20000.20 at $\phi=168^{\circ}$. This is the phase where spanwise rollers deform into $\Lambda$-shaped vortices.

This is one of the distinct characteristic of $K$-type secondary instability (Kachanov, Kozlov \& Levchenko 1977). It should be noted that these rollers are formed from the bottom wall up to $y=10$. By further examining the power spectrum of streamwise velocity fluctuations at $\phi=138^{\circ}$ calculated over the $y=1$ plane, we observe that the energy is stored in two major wavelengths: $\kappa_{x}=6$ and $\kappa_{x}=12$ (see figure 14). The wavenumber $\kappa_{x}=6$ corresponds to the shortest distance between two consecutive rollers. The interaction between these two wavelengths, as the ratio of the first one to the second is $1 / 2$, clearly characterizes the $K$-type secondary instability. At $R e_{\delta}=2500$, on the other hand, instead of the $\Lambda$-shaped vortex series, the rollers are lifted up to $y=20$. As these rollers are energized, they distort the flow around them and then were surrounded by rib vortices (see figure 5). This observation is similar to the characteristics of instability in free shear layers. It should be emphasized that the energy in the coherent vortices seems to play an important role in the breakdown process. For $\operatorname{Re}_{\delta}=2500$, at the moment when the rib vortices occur, the velocity profile become distinctly different from the laminar profile and r.m.s. velocity fluctuations become more isotropic. On the other hand, at $R e_{\delta}=2000$ the velocity profile follows the laminar profile when the breakdown, or secondary instability, is initiated. Noticeable deviation from the laminar profile is observed only after the nonlinear growth is initiated. Although both observations take place at deceleration, the first path suggests the influence of viscous effects in the breakdown process. In summary, the generated rollers follow two different paths during their breakdown into turbulence. The breakdown characteristics of the rollers require more in-depth hydrodynamic stability analyses.

Regardless of the breakdown process, for a flow to be characterized as turbulent, the resultant flow structures should display appropriate turbulent structures and statistics. Most importantly, the cascade of energy from the energy-containing wavelengths of the linear or weakly nonlinear instabilities to the smaller dissipative scales should be complete. In other words, the energy spectra from the large energetic scales to the Kolmogorov scale should follow inertial and dissipative scaling. In our simulations, we 
observe that the nonlinear stage of the instability and the breakdown of the rollers take place at the deceleration phases. If the breakdown process is complete, flow remains turbulent until the end of the wave period $\left(\phi=180^{\circ}\right)$. By making use of this observation, we examine whether the flow is transitional or fully turbulent by analysing the power spectrum of the fluctuating velocity components at the end of the wave period $\left(\phi=180^{\circ}\right)$. If the energy spectra at the end of the wave period follow classical turbulent scaling, then the flow is considered to be turbulent. This is illustrated in figure 15 where the power spectra of selected simulations are shown (for cases R1500-0.20, R2000-0.20, R2500-0.01, and R4000-0.01).

The spectra of R1500-0.20 and R4000-0.01 are first contrasted here due to the fact that at $R e_{\delta}=1500$ only the spanwise rollers are present at the end of the wave period, while at $R e_{\delta}=4000$, a developed turbulence is achieved. At $R e_{\delta}=1500$ (figure 15a), most of the energy in streamwise velocity fluctuations is concentrated in the $\kappa_{x}=4$ mode, which corresponds to the shortest distance between two consecutive rollers in the streamwise direction. On the other hand, a broad energy spectrum with a limited inertial range of $-5 / 3$ decay rate is observed for $\mathrm{R} 4000-0.01$, which is indicative of fully developed turbulence. At $R e_{\delta}=2000$ (figure $15 b$ ), although we observe chaotic motion at the end of the wave period after the breakdown of the coherent spanwise vortices, the wavelength $\kappa_{x}=6$ still shows a distinguishable peak and only a weak cascade of energy to the higher wavenumbers can be observed. Based on the $\log -\log$ plot of the spectrum, at $R e_{\delta}=2000$ inertial and dissipative ranges cannot be clearly identified. For $R e_{\delta}=2500$ (figure $15 c$ ), we can observe the effect of the energy transfer of the fluctuating velocities from the smaller wavelengths, where initial linear or weakly nonlinear instabilities are observed, to dissipative scales of higher wavenumber. Therefore, we can conclude that cases R2500-0.01 and R40000.01 can be categorized as turbulent, while R2000-0.2 falls into the transitional regime.

\subsection{Turbulent kinetic energy budget}

The characteristics of the fluctuating velocity components that we have observed in the power spectra are consistent with the time series of the turbulent kinetic energy (TKE) budget. The integral (over the volume of the entire domain, $\forall$ ) form of the TKE budget is given as follows:

$$
\frac{\partial k}{\partial t}=P-\epsilon,
$$

where $k$ represents the volume integral of turbulent kinetic energy

$$
k=\int \frac{1}{2} U_{i}^{\prime} U_{i}^{\prime} \mathrm{d} \forall,
$$

$P$ represents the production term

$$
P=\int-U_{i}^{\prime} U_{j}^{\prime} \frac{\partial\left\langle U_{i}\right\rangle}{\partial x_{j}} \mathrm{~d} \forall,
$$

where $\langle\cdot\rangle$ represents the plane-averaging over $x-z$ planes at each vertical point, and

$$
\epsilon=\frac{1}{R e_{\delta}} \int \frac{\partial U_{i}^{\prime}}{\partial x_{j}} \frac{\partial U_{i}^{\prime}}{\partial x_{j}} \mathrm{~d} \forall
$$

represents dissipation. The time series of the TKE budget terms calculated from simulations R2000-0.02, R2500-0.01, and R4000-0.01 are given in figure 16. The 
(a)

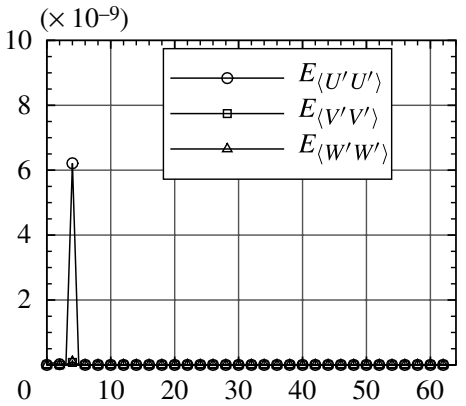

(b)

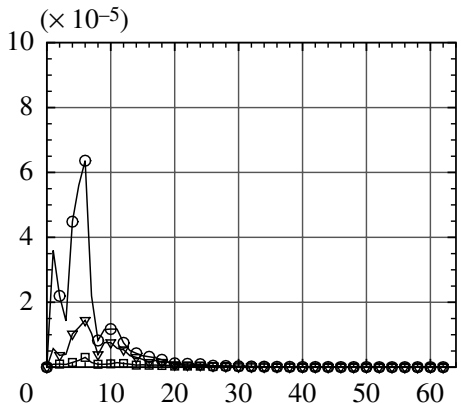

(c)

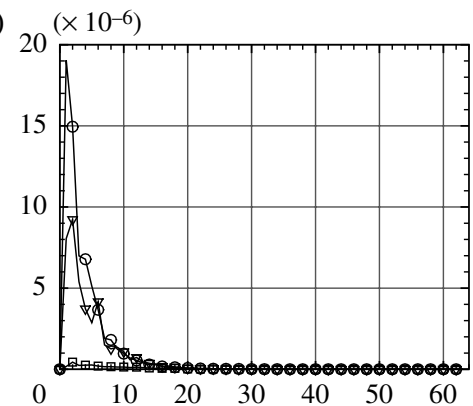

(d)

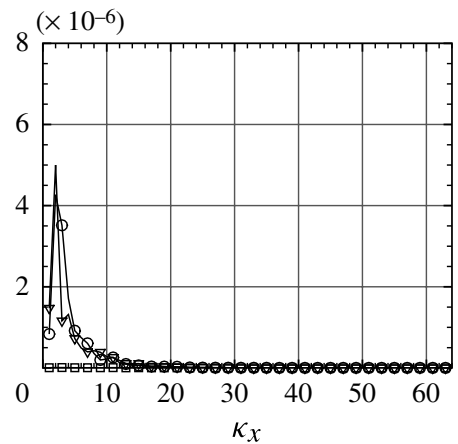

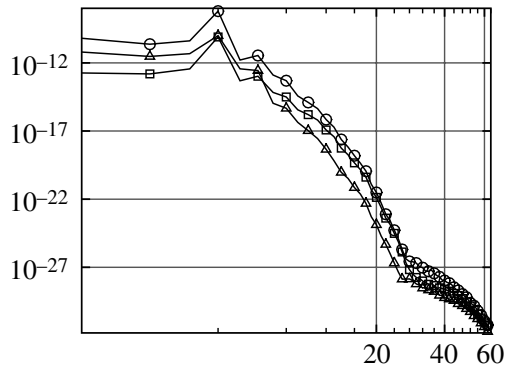
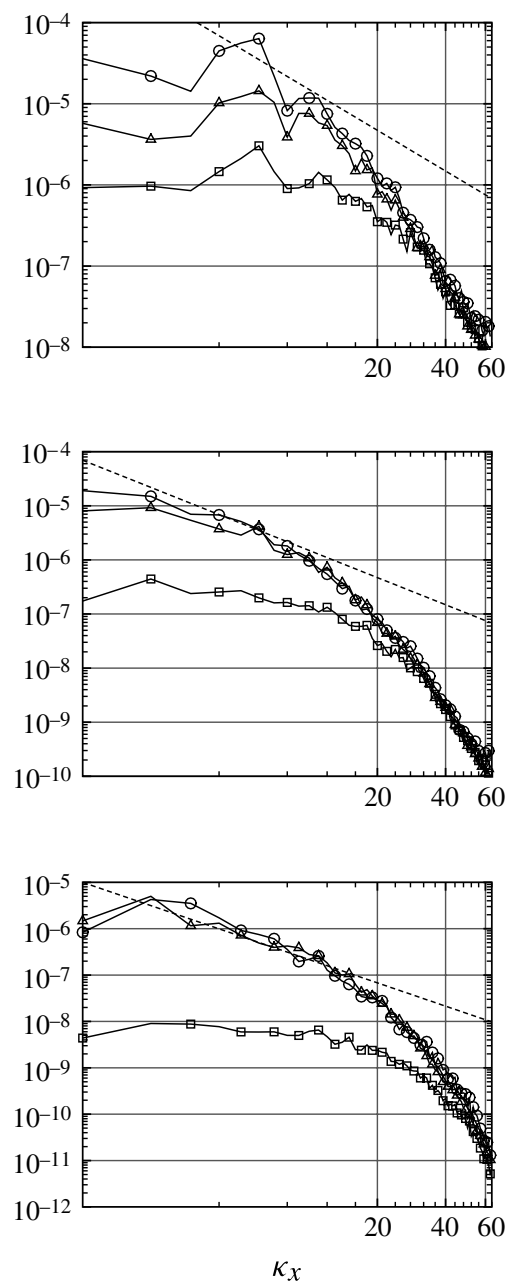

FIGURE 15. Power spectra of the fluctuating velocity component, at $y=0.5$, in the flow direction at: (a) $R e_{\delta}=1500(\mathrm{R} 1500-0.20) ;(b) R e_{\delta}=2000(\mathrm{R} 2000-0.20) ;(c) R e_{\delta}=2500$ (R2500-0.01); and $(d) R e_{\delta}=4000$ (R4000-0.01). On the left are linear plots of the wavelength versus the spectrum in the flow direction to show the energy spectrum distribution in the leading wavelengths. The plots on the right are $\log -\log$ versions to show whether the energy cascade in a developed turbulence is reached. In the $\log -\log$ plots $-5 / 3$ slope is shown for reference. 
(a)

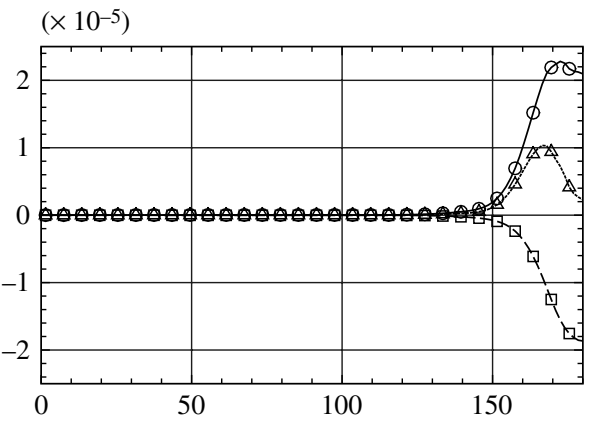

(b)

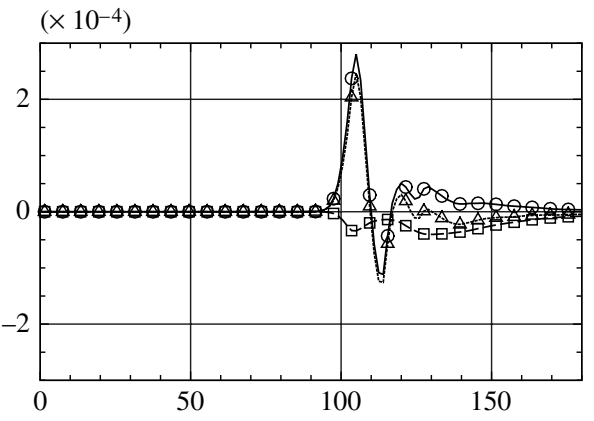

(c)

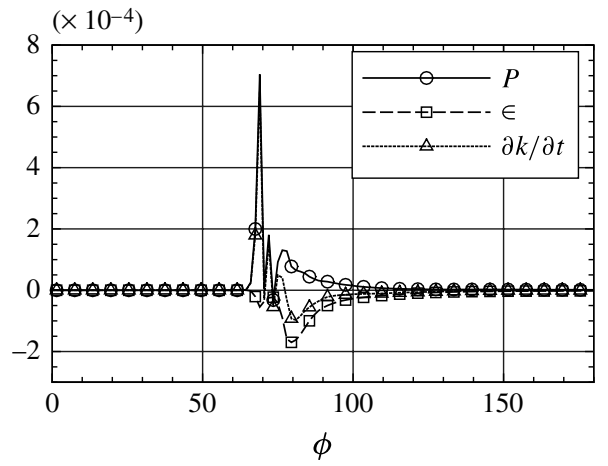

FIGURE 16. Time series of integral production, dissipation and time rate of change in the integral TKE for the simulations: (a) R2000-0.01; (b) R2500-0.01; and (c) R4000-0.01.

formation of the fully developed turbulent energy cascade from smaller wavelengths to the dissipative scales that is observed from the power spectra is correlated with the growth of the volume-integrated turbulent dissipation term given in $(4.1 d)$. In all the three cases shown, with the initiation of the nonlinear growth in velocity fluctuations, the production term rapidly increases. However, it takes a longer time for the cascade of fluctuations to be completed, and therefore there exists a time-lag associated with the increase of the magnitude in the time series of turbulent dissipation. To balance the budget, the term $\partial k / \partial t$ hence first increases together with the increase in production. The absolute value of $\partial k / \partial t$ starts decaying after the magnitude of turbulent dissipation starts to increase. This is clearly observed from the simulation results of R2500-0.01 and R4000-0.01 (figure 16b,c). Also, during the later part of the wave period in the decelerating phase, we observe the classic feature of boundary 
layer turbulence where production is balanced by dissipation. On the other hand, for R2000-0.20 (see figure 16a), turbulent dissipation does not reach its peak even at the end of the wave period. Therefore, it can also be concluded from the times series of TKE budget terms that the flow in case R2000-0.20 remains transitional even at $\phi=180^{\circ}$.

\subsection{Classification of the flow regimes}

By making use of the analyses discussed in the previous sections, including coherent vortical structures, flow statistics, energy spectra of velocity fluctuations and integral TKE budget, we confirm the following four flow regimes of the bottom boundary layer under isolated solitary wave motion (see also table 1).

(a) Laminar regime $\left(R e_{\delta}<400\right)$ : In this regime, there is no sign of instability as there is no growth of r.m.s. velocity fluctuations within the boundary layer. Prescribed initial random perturbation always decays and the base flow remains laminar.

(b) Disturbed laminar regime $\left(400<R e_{\delta}<1500\right)$ : The linear instability prevails for a portion of the decelerating phase of the wave period. Formation of roller pairs is observed as a result of this instability. Depending on the amplitude of background disturbance and Reynolds number, a weak long wave and viscous nonlinear instability may be present during the acceleration phase. However, neither instability mechanisms is strong enough to alter the laminar base flow and there is no sign of transitional or turbulent fluid motion.

(c) Transitional regime $\left(1500<R e_{\delta} \lesssim 2400\right)$ : In this regime, the formation of the rollers at deceleration is followed by strong nonlinear growth and breakdown processes and the base flow deviates from a laminar velocity profile. However, the breakdown process is not completed and the dissipative scales of turbulence are not completely formed. In most cases, the short-lived long-wave viscous nonlinear instability is present; however, this mechanism is not the major one that leads to turbulence.

(d) Turbulent regime $\left(\approx 2400<R e_{\delta}\right)$ : In this regime, the breakdown of the coherent vortical structures into fully developed turbulence is complete and the dissipative scales are completely formed within the wave period.

It must be stressed that the ranges of Reynolds numbers given above to differentiate the flow regimes are somewhat affected by the viscous nonlinear instability that occurs during the acceleration phase. This instability influences the level of background velocity fluctuation for the rest of the wave period and thus affects the nonlinear growth of the velocity fluctuations at deceleration and the formation of fully developed turbulence. For example, based on the present criterion for the classification of the flows, at $R e_{\delta}=2200$, cases R2200-0.01, R2200-0.02 and R2200-0.05 remain transitional, while cases R2200-0.10 and R2200-0.20 can be categorized as turbulent. Namely, turbulent regime is reached for higher values of $\varepsilon$ at this Reynolds number. Above $\operatorname{Re}_{\delta}=2400$, all the simulations become turbulent regardless of $\varepsilon$. Therefore, we set the lower limit of the turbulent regime to be at $R e_{\delta}=2400$. More details can be found in table 1 .

\section{Conclusions}

In this study, the instability mechanisms and the characteristics of flow turbulence under an isolated solitary wave are investigated through 70 direct numerical simulations. The initial condition for the fluctuating velocity field is specified to 
be random and at each Reynolds number five different levels of initial disturbance amplitude are considered.

Through a depth- and time-dependent spectral analysis, we have identified two instability mechanisms to be present. The first one is a viscous, nonlinear, and long-wave instability that takes place at the acceleration phase, if the background disturbance is of sufficient amplitude. The second one is a broadband instability that occurs during the deceleration phase. The second instability is found to be the one that leads to chaotic motion and eventual transition to turbulence. Although the adverse pressure gradient and the presence of inflection points in the mean flow suggest a possible inviscid origin for this instability, caution should be exercised. For example, in the nonlinear secondary instability at $R e_{\delta}=2000$, the influence of viscosity is observed.

The growth of linear instability towards nonlinear chaotic motion and transition to turbulence follows two different paths: (i) $K$-type secondary instability which is characterized by the formation of aligned $\Lambda$-shaped vortices; and (ii) breakdown similar to that in free shear layers characterized by the formation of rib vortices around energized spanwise rollers. While the first one is observed for a narrow range of $R e_{\delta}$ between 1500 and 2000, the second one is observed for $R e_{\delta}>2000$. The implication of the two different pathways to chaotic motion is summarized as follows. $K$-type secondary instability suggests a viscous origin of the underlying primary linear instability. With the increase in $R e_{\delta}$, viscosity appears to lose its significance and the path towards chaotic motion becomes more like the ones observed in free shear layers. This conjecture requires in-depth hydrodynamic stability analyses.

As a result of these simulations, we confirm the four different flow regimes. The first regime is the laminar regime where the initial velocity fluctuations continually decay and no deviation from the laminar mean velocity profile is observed in the whole wave period. The second regime is the disturbed laminar regime. In this regime, linear instability, observed at deceleration, is dominant; however the level of the fluctuations is not strong enough to alter the mean velocity profile. The third regime is the transitional regime, in which nonlinear growth of the fluctuations is observed. However, the transition process is not complete within a wave period and a fully developed turbulent state is not observed. The fourth regime is the turbulent regime in which the transition process is complete within the wave period and a wide range of turbulent scales from the largest to the dissipative scales are formed.

Here we follow the work of Grimshaw (1971), Liu \& Orfilia (2004) and Vittori \& Blondeaux (2011) to consider transition and turbulence in the bottom boundary layer of a solitary wave of elevation. A similar investigation of flow and instability due to a solitary wave of depression can be carried out as well (see Lin \& Rodekopp 2011). Unlike the solitary wave of elevation, the amplitude of the depression waves becomes wider with the increase in the degree of nonlinearity. Also, with the increase in the degree of nonlinearity the velocity difference across the boundary layer increases. Therefore, more favourable conditions for flow instability and turbulence generation are present for depression waves compared to solitary wave of elevation. However, here we restrict attention to the limit where the ratio of wave height to layer height is very small. In this limit, the differences between solitary waves of elevation and depression can be expected to be small. The instability mechanisms and boundary layer turbulence for flows driven by solitary wave of depression requires further investigation. 


\section{Acknowledgements}

This study is supported by National Science Foundation (CMMI-1135026; OCE1130217; OCE-1131016). Simulations presented in this paper are carried out on CHIMERA at the University of Delaware with funding supported by the National Science Foundation (CNS-0958512). Simulations also leverage computing resource provided by Extreme Science and Engineering Discovery Environment (XSEDE) (TGOCE100015).

\section{REFERENCES}

Apel, J. R., Byrne, H. M., Proni, J. R. \& Charnell, R. L. 1975 Observations of oceanic internal and surface waves from the earth resources technology satellite. J. Geophys. Res. 80 (6), 865-881.

Blondeaux, P. \& VitToRi, G. 2012 RANS modelling of the turbulent boundary layer under a solitary wave. Coast. Engng 60, 1-10.

Blondeaux, P., Pralits, J. \& Vittori, G. 2012 Transition to turbulence at the bottom of a solitary wave. J. Fluid Mech. 709, 396-407.

Bogucki, D. J., Rodekopp, L. G. \& BARTH, J. 2005 Internal solitary waves in the coastal mixing and optics 1996 experiment: multimodal structure and resuspension. J. Geophys. Res. 110, C02024.

Cantero, M. I., Balachandar, S. \& Garcia, M. 2008 An Eulerian-Eulerian model for gravity currents driven by inertial particles. Intl J. Multiphase Flow 34, 484-501.

Canuto, C., Hussaini, M. Y., Quarteroni, A. \& Zang, T. A. 1987 Spectral Methods in Fluid Dynamics. Springer.

Cortese, T. \& BALACHANDAR, S. 1995 High performance spectral simulation of turbulent flows in massively parallel machines with distributed memory. Intl J. Supercomput. Appl. 9 (3), 187-204.

Dawson, A. G. \& ShI, S. 2000 Tsunami deposits. Pure Appl. Geophys. 157, 875-897.

DiAmessis, P. J. \& Rodekopp, L. G. 2006 Numerical investigation of solitary internal wave-induced global instability in shallow water benthic boundary layers. J. Phys. Oceanogr. 36, 784-812.

Drazin, P. G. \& ReID, W. H. 1981 Hydrodynamic Stability. Cambridge University Press.

Grimshaw, R. 1971 The solitary wave in water of variable depth. J. Fluid Mech. 46, 611-622.

Kachanov, Y. S., Kozlov, V. V. \& Levchenko, V. Y. 1977 Nonlinear development of a wave in a boundary layer. Fluid Dyn. 12, 383-390.

Kim, J., Moin, P. \& Moser, R. 1987 Turbulence statistics in fully developed channel flow at low Reynolds number. J. Fluid Mech. 177, 133-166.

Lin, Y. \& Rodekopp, L. G. 2011 The wave-induced boundary layer under long internal waves. Ocean Dyn. 61, 1045-1065.

LiU, P. L.-F. \& ORFILIA, A. 2004 Viscous effects on transient long-wave propagation. J. Fluid Mech. 520, 83-92.

LiU, P. L.-F., PARK, Y. S. \& Cowen, E. A. 2007 Boundary layer flow and bed shear stress under a solitary wave. J. Fluid Mech. 574, 449-463.

Negretti, M. E. \& Socolofsky, S. A. 2005 Stabilization of cylinder wakes in shallow water flows by means of roughness elements: an experimental study. Exp. Fluids 38, 403-414.

SEOL, D. G. \& JIRKA, G. H. 2010 Quasi-tow dimensional properties of single shallow vortex with high initial Reynolds numbers. J. Fluid Mech. 665, 274-299.

Son, S., LynetT, P. J. \& KIM, D.-H. 2011 Nested and multi-physics modelling of tsunami evolution from generation to inundation. Ocean Model. 38 (1-2), 96-113.

SPALART, \& BALDWIN, 1989 Direct simulation of a turbulent oscillating boundary layer. In Turbulent Shear Flows 6 (ed. J. C. Andre), pp. 417-440. Springer.

StastnA, M. \& LAMB, K. G. 2002 Vortex shedding and sediment resuspension associated with the interaction of an internal solitary wave and the bottom boundary layer. Geophys. Res. Lett. 29 (11), 7-1-7-3. 
Sumer, B. M., Jensen, P. M., Søerensen, L. B., Fredsøe, J. \& LiU, P. L. F. 2010 Coherent structures in wave boundary layers. Part 2. Solitary motion. J. Fluid Mech. 646, 207-231.

Synolakis, C. E., E. N., Bernard, Titov, V. V., KÂnoĞlu, U. \& GonzÁlez, F. I. 2008 Validation and verification of tsunami numerical models. Pure Appl. Geophys. 165 (11-12), 2197-2228.

Tonkin, S., Yeh, H., Kato, F. \& Sato, S. 2003 Tsunami scour around a cylinder. J. Fluid Mech. 496, 165-192.

Vittori, G. \& Blondeaux, P. 2008 Turbulent boundary layer under a solitary wave. J. Fluid Mech. 615, 433-443.

Vittori, G. \& Blondeaux, P. 2011 Characteristics of the boundary layer at the bottom of a solitary wave. Coast. Engng 58, 206-213.

Voit, S. S. 1987 Tsunamis. Annu. Rev. Fluid Mech. 19, 217-236.

Zhou, J., Adrian, R. J., Balachandar, S. \& Kendall, T. M. 1999 Mechanisms for generating coherent packets of hairpin vortices in channel flow. J. Fluid Mech. 387, 353-396. 